\title{
ASYMPTOTIC EXPANSIONS FOR POLYNOMIALS ORTHOGONAL WITH RESPECT TO A COMPLEX NON-CONSTANT WEIGHT FUNCTION
}

\author{
A. APTEKAREV AND R. KHABIBULLIN
}

\begin{abstract}
We consider a sequence of polynomials that are orthogonal with respect to a complex analytic weight function which depends on the index $n$ of the polynomial. For such polynomials we obtain an asymptotic expansion in $1 / n$. As an example, we present the asymptotic expansion for Laguerre polynomials with a weight that depends on the index of the polynomial.
\end{abstract}

\section{INTRODUCTION}

1.1. The setting and the formulation of the problem. Let $Q_{j} \in H(\Omega), j=0,1$, be holomorphic functions in a domain $\Omega$ which define a sequence of weight functions,

$$
W_{n}(z):=e^{-2 n\left(Q_{0}(z)+\frac{1}{n} Q_{1}(z)\right)},
$$

considered in a domain $\Omega$ that contains a smooth Jordan $\operatorname{arc} F$, and let

$$
\left\{P_{k}(z)=z^{k}+\cdots\right\}_{k=0}^{\infty}: \quad \int_{F} P_{n}(z) z^{\nu} W_{n}(z) d z=0, \quad \nu=0, \ldots, n-1,
$$

be polynomials which are orthogonal along the contour $F$ in the complex plane with respect to the non-constant weight $W_{n}(z)$.

In the present work we study the asymptotic behaviour of the polynomials $P_{n}$ as $n \rightarrow \infty$. The goal is to obtain a complete asymptotic expansion in powers of $1 / n$.

An explicit form of the asymptotics of (21), (11) makes it possible to solve a number of important problems in diverse areas of mathematics. In [1, 2], they were used to determine the rate of approximation of analytic functions by rational functions. Among many other impressive applications we note universality theorems in the theory of random matrices [3, 4, 5], applications to asymptotic combinatorics [6, 7] and mathematical physics [8, 9 .

The foundations of the asymptotic theory of orthogonal polynomials was laid by S. N. Bernstein [10] and G. Szegö [11] (in the case of a real weight on an interval in the real line). Bernstein's method allows an extension to complex-valued functions [12, 13, 14, 15]. Stahl [16] explored the connection between the asymptotics of complex-valued orthogonal polynomials and extremal problems in the theory of logarithmic potential in the plane. Significant progress in the asymptotic theory of orthogonal polynomials has been achieved recently by using methods of the matrix Riemann-Hilbert problem. Its, Kitaev, and Fokas (see [8, 17]) suggested a reformulation of the orthogonality conditions

2000 Mathematics Subject Classification. Primary 42C05.

This work has been supported by the Russian Fund for Fundamental Research (grant No. 0501-00522), the Support of Leading Scientific Institutions in the RF Programme (grant No. NSh1551.2003.1), The Mathematical Sciences Department of the Russian Academy of Sciences (programme no. 1) and the INTAS fund (grant No. 03-516637). 
for (2) in terms of a matrix Riemann-Hilbert problem. The steepest descent method for an asymptotic solution of this problem was suggested by Deift and Zhou [18. The matrix Riemann-Hilbert problem method has not only made it possible to make considerable progress in the asymptotic theory of polynomials orthogonal with respect to a non-constant real-valued weight function (see [3, 4, 19, 20, 21]), but also proved capable of being extended to the case of complex-valued non-constant weights in the complex plane [2, 6, 9]. Apart from the above references, a detailed description of this method is contained in the monograph 22]; an approachable exposition of the main ideas can also be found in 23 .

One of the merits of the Deift-Zhou steepest descent method is that it not only allows us to obtain an explicit expression for the leading term of the asymptotic series of the polynomials $P_{n}$, but also enables us to describe the entire asymptotic expansion. This has been shown in [3, 20, which present the asymptotic expansion of the polynomials (2) with

$$
F:=\mathbb{R}, \quad Q_{1}=0, \quad Q_{0}(z)=\sum_{j=0}^{2 m} q_{j} z^{j}, \quad q_{j} \in \mathbb{R}, \quad q_{2 m}>0 .
$$

The paper 24] contains a more refined description of the asymptotics of [3, 20]; in particular, explicit formulae are obtained for the coefficients of the expansion in powers of $\frac{1}{n}$. Let us also note the paper [25], which studies the asymptotics in the case of

$$
F:=[-1,1], \quad W_{n}(z)=W(z)(z-1)^{\alpha}(z+1)^{\beta}, \quad Q_{0}=0,
$$

where $W(z)$ is a real analytic function on $[-1,1]$.

In the present work we describe the asymptotic expansions for polynomials (2) under sufficiently general conditions on the complex-valued functions $Q_{0}$ and $Q_{1}$, which ensure that a contour $F$ can be chosen to be a connected arc in the complex plane. Thus we extend the results of [2, where under similar conditions the leading term of the asymptotic expansion of $P_{n}$ was obtained. At the same time, in our constructions and proofs we make essential use of the ideas and techniques introduced in the study of asymptotic expansions in [3, 24, 25].

1.2. Statement of the results. To formulate the main theorem we will need the following concepts.

1) An important rôle is played by the extremal measure $\lambda$ which ensures the equilibrium of the logarithmic potential

$$
V_{\nu}(z):=\int_{F} \ln \frac{1}{|t-z|} d \nu(t)
$$

in the external field $f:=\mathfrak{R}\left(Q_{0}\right)$ :

$$
V_{\lambda}+f \begin{cases}=\gamma_{\Delta} & \text { on } S(\lambda), \\ \geq \gamma_{\Delta} & \text { on } F .\end{cases}
$$

We take the support $S(\lambda)$ of this measure to be a connected arc, $S(\lambda)=: \Delta=: \overline{[a, b]}$, connecting the points $a$ and $b$. We fix the orientation to be from $a$ to $b$ so that the limiting values of functions in the approach to $\Delta$ from the left (right) will be indicated by $+(-)$. that

If the measure $\lambda$ (which always exists and is unique) satisfies the additional property

$$
\frac{\partial\left(V_{\lambda}+f\right)}{\partial n_{+}}=\frac{\partial\left(V_{\lambda}+f\right)}{\partial n_{-}} \quad \text { on } \quad S(\lambda)
$$

then we say that the pair $(F, f)$ has the $S$-property; we will denote this fact by $(F, f) \in S$. 
2) In addition, the expression for the asymptotics will contain the so-called Szegö function $\varphi_{n}$ for the weight function $W$, which is defined as the solution of the following boundary value problem:

$$
\begin{gathered}
\varphi_{n} \in H(\mathbb{C} \backslash \Delta), \quad \varphi_{n} \neq 0 \quad \text { in } \mathbb{C} \backslash \Delta, \quad \exists \varphi_{n \pm} \in L^{2}(\Delta), \\
\varphi_{n+} \varphi_{n-} W=1 \quad \text { in } \Delta, \\
\varphi_{n}(z)=\frac{z^{n}}{C_{n}}+\cdots, \quad z \rightarrow \infty
\end{gathered}
$$

A solution of the problem (5) exists and is unique (up to sign \pm ) for some $C_{n}$ determined from $W$. Properties of this solution and its explicit form will be discussed later (see Subsection 2.2).

The main result of the present work is the following theorem, which provides a complete asymptotic expansion for the polynomials $P_{n}$ outside the arc $\Delta$ of the support of the equilibrium measure $\lambda$.

Theorem 1. Let the sequence of analytic weight functions $\left\{W_{n}\right\}_{n=0}^{\infty}$ be given by (11) and let the smooth Jordan arc F satisfy the following conditions:

1) $\left(F, \mathfrak{R}\left(Q_{0}\right)\right) \in S$, the equilibtrium set on $F$

$$
\Delta:=\left\{z: V_{\lambda}(z)+\mathfrak{R}\left(Q_{0}(z)\right)=\gamma_{\Delta}\right\}
$$

is connected, and $\lambda^{\prime}>0$ at all interior points of the arc $\Delta$.

2) The behaviour of the derivative $\lambda^{\prime}$ of the equilibrium measure (in the field $Q_{0}$ ) in a neighbourhood of the endpoints $a$ and $b$ of the support $\Delta$ is described by

$\lambda^{\prime}(z):=((z-a)(z-b))^{1 / 2} h(z), \quad$ for $\quad h \in H\left(O_{a} \cup O_{b}\right), \quad z \in O_{a} \cup O_{b}$.

Then

A) for sufficiently large $n$ there are polynomials $P_{n}(z)=z^{n}+\cdots$, defined by the condition (2), that are orthogonal with weights $W_{n}$ on $F$;

B) For $z \in \mathbb{C} \backslash \Delta$, the expression $P_{n}(z) / C_{n} \varphi_{n}(z)$, with a Szegö function $\varphi_{n}$ (for the weight function $W_{n}$ ), defined in (5), has an asymptotic expansion in powers of $1 / n$ of the form

$$
\frac{P_{n}(z)}{C_{n} \varphi_{n}(z)} \sim \frac{1}{2}\left(\beta(z)+\beta^{-1}(z)\right)\left[1+\sum_{k=1}^{\infty} \frac{\Pi_{k}(z)}{n^{k}}\right] \quad \text { as } \quad n \rightarrow \infty,
$$

where

$$
\beta(z)=\left(\frac{z-b}{z-a}\right)^{1 / 4}
$$

This expansion is uniform in $z$ for compact sets in $\overline{\mathbb{C}} \backslash \Delta$. The functions $\Pi_{k}$ are analytic in $\mathbb{C} \backslash \Delta$ and can be computed explicitly.

The system of boundary value problems that defines the coefficients $\Pi_{k}$ recurrently will be given in Subsections 2.9 and 2.10. Here we only quote the explicit form of the 
coefficient of $1 / n$ in the expansion (6):

(8)

$$
\begin{aligned}
\Pi_{1}(z)= & \frac{i}{48 h(b)(z-b)}\left\{-\frac{5}{(z-b)}+\frac{6}{(b-a)}+\frac{3 h^{\prime}(b)}{h(b)}\right\}+i c_{b}^{2} \frac{(b-a)}{16 h(b)} \\
& +\frac{i}{48 h(a)(z-a)}\left\{\frac{5}{(z-a)}-\frac{6}{(a-b)}-\frac{3 h^{\prime}(a)}{h(a)}\right\}-i c_{a}^{2} \frac{(a-b)}{16 h(a)} \\
& +i \frac{\beta(z)-\beta^{-1}(z)}{\beta(z)+\beta^{-1}(z)}\left[\frac{1}{48 h(b)(z-b)}\left\{-\frac{5}{(z-b)}-\frac{8}{(b-a)}+\frac{3 h^{\prime}(b)}{h(b)}\right\}\right. \\
& \left.-c_{b}^{2} \frac{(b-a)}{16 h(b)}+\frac{1}{48 h(a)(z-a)}\left\{-\frac{5}{(z-a)}-\frac{8}{(a-b)}+\frac{3 h^{\prime}(a)}{h(a)}\right\}-c_{a}^{2} \frac{(a-b)}{16 h(a)}\right]
\end{aligned}
$$

where the coefficients $c_{b}$ and $c_{a}$ are given by the formulae

$$
c_{\alpha}:=\frac{1}{2 \pi i} \int_{\gamma} \frac{-2 Q_{1}(\zeta)}{((\zeta-a)(\zeta-b))^{1 / 2}} \frac{d \zeta}{(\zeta-\alpha)}, \quad \alpha=a, b,
$$

respectively, and the integral is taken along a closed contour $\gamma$ that encloses the support of the equilibrium measure $\lambda$ and is oriented counterclockwise.

The proof of Theorem 1 is presented in the next section. In $\S 3$ we obtain the explicit form of the coefficient $\Pi_{1}(z)$ (that is, (8), (91)). Finally, in $\S 4$ we consider an example of an asymptotic expansion in $n$ for the Laguerre polynomials $L_{n}^{\left(-A n-A_{1}\right)}(x)$. A different example, which uses Theorem 1 to obtain an asymptotic expansion for Bessel polynomials, was considered in [26].

\section{The method of the Riemann-Hilbert matrix Problem FOR OBTAINING STRONG ASYMPTOTICS OF ORTHOGONAL POLYNOMIALS}

2.1. The Riemann-Hilbert matrix problem for orthogonal polynomials. In addition to polynomials $P_{n}(z)=z^{n}+\cdots$, we will also consider functions of the second kind,

$$
R_{n}(z):=\frac{1}{2 \pi i} \int_{F} \frac{P_{n}(t) W_{n}(t)}{t-z} d t, \quad R_{n}(z)=O\left(\frac{1}{z^{n+1}}\right) .
$$

Together with the pair $P_{n}, R_{n}$, we will consider pairs $\tilde{P}_{n-1}, \tilde{R}_{n-1}$ such that

$$
\begin{aligned}
& \int_{F} \tilde{P}_{n-1}(z) z^{\nu} W_{n}(z) d z=0, \quad \nu=0, \ldots, n-2, \quad \tilde{P}_{n-1}(z)=z^{n-1}+\cdots, \\
& \tilde{R}_{n-1}(z):=\frac{1}{2 \pi i} \int_{F} \frac{\tilde{P}_{n-1}(t) W_{n}(t)}{t-z} d t, \quad \tilde{R}_{n-1}(z)=\frac{1}{m z^{n}}+\cdots, \quad z \rightarrow \infty,
\end{aligned}
$$

where $m$ is some constant that depends on $n$.

Following [8], we consider matrix-valued functions

$$
Y:=\left(\begin{array}{cc}
P_{n} & R_{n} \\
m \tilde{P}_{n-1} & m \tilde{R}_{n-1}
\end{array}\right), \quad \mathbf{W}:=\left(\begin{array}{cc}
1 & W_{n} \\
0 & 1
\end{array}\right) .
$$

The function $Y \in H_{2 \times 2}(\mathbb{C} \backslash F)$ has continuous (non-tangential) boundary values $Y_{+}$ and $Y_{-}$"inside" $F$ (that is, with the exception of the boundary points), and these boundary values are $L^{2}$-integrable on the whole of $F$.

From the definition (10) of $R_{n}$, as well as from the definition of $\tilde{R}_{n-1}$, in view of the Sokhotskii-Plemelj relation, functions of the second kind satisfy the boundary relations

$$
P_{n} W_{n}=R_{n+}-R_{n-}, \quad \tilde{P}_{n-1} W_{n}=\tilde{R}_{n-1_{+}}-\tilde{R}_{n-1_{-}} .
$$


Therefore the function $Y$ is a solution of the following matrix Riemann-Hilbert boundary value problem:

$$
\begin{gathered}
Y \in H_{2 \times 2}(\mathbb{C} \backslash F), \quad \exists Y_{ \pm} \in L_{2 \times 2}^{2}(F), \\
Y_{+}=Y_{-} \mathbf{W} \quad \text { "inside" } \quad F, \\
Y(z)=\left(I+O\left(\frac{1}{z}\right)\right)\left(\begin{array}{cc}
z^{n} & 0 \\
0 & z^{-n}
\end{array}\right), \quad z \rightarrow \infty .
\end{gathered}
$$

The boundary value problem (11) is identically equivalent to the definition of the orthogonal polynomials (2); its solution (once we fix the behaviour at the endpoints of $F$ ) is unique (see [22]).

The main idea of the method (see [22]) consists in effecting the consecutive transformations $Y \rightarrow T \rightarrow S \rightarrow R$ of the original Riemann boundary value problem to obtain an equivalent problem that is normalized at infinity and has a jump matrix that tends uniformly to the identity matrix as $n \rightarrow \infty$. Using the fact that for $R$ the jump matrix has a complete asymptotic expansion in powers of $1 / n$, we will obtain a complete asymptotic expansion for $R$ itself. Therefore, reversing the direction in the sequence of transformations $Y \rightarrow T \rightarrow S \rightarrow R$, we will obtain complete asymptotic expansions for the matrix $Y$ and thus for the required orthogonal polynomials together with the related functions of the second kind.

- The first transformation $Y \rightarrow T$, which uses the Szegö function for the restriction of the weight function $W_{n}$ to the support $\Delta \subseteq F$ of the equilibrium measure (4) in the field $\mathfrak{R}\left(Q_{0}\right)$, allows us to pass to a boundary value problem the solution of which (unlike $Y$ ) is a function that is holomorphic in $\overline{\mathbb{C}} \backslash F$, that is, without singularities at infinity, while the jump matrix allows a factorization in $\Delta$ which we will find very convenient.

- The second transformation $T \rightarrow S$ includes a factorization of the jump matrix for $T$ with a further deformation of the contour $F$ ("opening up the lens") and an equivalent transformation from a boundary value problem for $T$ on the contour $F$ to one for $S$ on the deformed contour $\Sigma_{1}$. As a result, at all the interior points of the contour $\Sigma_{1} \backslash \Delta$ the jump matrix for $S$ converges as $n \rightarrow \infty$ to the identity matrix, while on $\Delta$ the jump is constant.

- Before effecting the third transformation $S \rightarrow R$, we consider two problems: first, to find a matrix $N$ with a constant jump on $\Delta$, and second, to find a matrix $U$ defined in neighbourhoods $O_{\delta}$ of the endpoints of the support of the equilibrium measure with a jump matrix which on the union of contours $O_{\delta} \cup \Sigma_{1}$ is identical to the jump matrix for $S$ on $\Sigma_{1}$.

- Finally, the third transformation $S \rightarrow R$ is a multiplication of $S$ by $N$ outside neighbourhoods of the endpoints and by $U$ inside these neighbourhoods. The matrix $R$ will also satisfy some Riemann boundary value problem. Due to the choice of the matrices $N$ and $U$ as well as the matching conditions, the jump matrix for $R$ will converge on the family $\Sigma$ of contours to the identity matrix as $n \rightarrow \infty$.

2.2. The equilibrium measure and the Szegö function. In this subsection we will discuss in more detail the concepts from the theory of logarithmic potential and the boundary value problems mentioned in the Introduction.

Let $M(F)$ be the set of positive probability measures $\nu$ on $F$ (that is, $S_{\nu}:=\operatorname{supp} \nu \subseteq$ $F)$ such that

$$
\int_{|t| \geq 1} \log |t| d \nu(t)<+\infty
$$


For each measure $\nu \in M(F)$ we define its logarithmic potential:

$$
V_{\nu}(z):=\int_{F} \ln \frac{1}{|t-z|} d \nu(t)
$$

Let us also consider two functionals on the space $M(F)$. By $I(\nu)$ we will denote the energy that does not take external fields into account:

$$
I(\nu)=\int V_{\nu} d \nu
$$

For any continuous function $f$ defined on $F$, we will denote by $I_{f}(\nu)$ the energy of the measure $\nu$ in the external field $f$ :

$$
I_{f}(\nu):=I(\nu)+2 \int_{F} f d \nu .
$$

There exists a unique measure $\lambda:=\lambda(F, f)$ that minimizes the energy functional $I_{f}$ (that is, $I_{f}(\lambda)=\inf _{\nu \in M(F)} I_{f}(\nu)$ ). This is the measure (see [11]) which satisfies the equilibrium condition (3).

Let us introduce the complex potential

$$
\mathcal{V}_{\lambda}(z):=V_{\lambda}(z)+i \tilde{V}_{\lambda}(z)=\int_{F} \ln \frac{1}{t-z} d \lambda .
$$

Here $\tilde{V}_{\lambda}$ is determined, modulo an imaginary constant, from the Cauchy-Riemann relations.

Let $\left(F, \mathfrak{R}\left(Q_{0}\right)\right) \in S$ (see (4) for a definition). Then the analyticity of $Q$ and (3) allow us to rewrite (4) as

$$
\frac{d}{d z}\left(\mathcal{V}_{\lambda+}+Q_{0}\right)=-\frac{d}{d z}\left(\mathcal{V}_{\lambda-}+Q_{0}\right) \text { on } \quad \Delta,
$$

which in turn entails the following boundary value problem for the complex potential:

$$
\mathcal{V}_{\lambda+}+\mathcal{V}_{\lambda-}=-2 Q_{0}+2 \Gamma
$$

where the uniquely determined real part of the constant $\Gamma$, in view of the equilibrium relation (3), is equal to the equilibrium constant

$$
\mathfrak{R}(\Gamma)=\gamma_{\Delta} .
$$

Therefore the function

$$
\Phi_{Q_{0}}:=\exp \left\{\Gamma-\mathcal{V}_{\lambda}\right\}
$$

satisfies the boundary condition

$$
\Phi_{Q_{0}+} \Phi_{Q_{0}-} e^{-2 Q_{0}}=1 \text { on } \Delta .
$$

It is known (see [28, p. 485]) that the boundary value problem

$$
\begin{gathered}
\tilde{s} \in H^{\infty}(\overline{\mathbb{C}} \backslash \Delta), \quad \tilde{s} \neq 0 \quad \text { in } \quad \overline{\mathbb{C}} \backslash \Delta, \\
\tilde{s}_{+} \tilde{s}_{-} \tilde{W}=1 \quad \text { on } \quad \Delta,
\end{gathered}
$$

has a unique solution (up to the sign \pm ) in the class of bounded functions; it is given by

$$
\tilde{s}(\tilde{W} ; z)=\exp \left\{w_{\Delta} \int_{\Delta} \frac{\log \tilde{W}(t)}{z-t} \frac{d t}{2 \pi i w_{\Delta+}}\right\},
$$

and furthermore

$$
\tilde{s}(\infty)=\exp \left\{\int_{\Delta} \frac{\log \tilde{W}(t) d t}{2 \pi i w_{\Delta+}}\right\} .
$$


Here

$$
w_{\Delta}(z)=((z-a)(z-b))^{1 / 2}, \quad \frac{w_{\Delta}(z)}{z} \rightarrow 1, \quad z \rightarrow 1 .
$$

Choosing $\tilde{W}:=e^{-2 Q_{1}}$ and taking into account (14) and (15), we obtain an explicit form for the Szegö function, the solution of the problem (5) with $W:=W_{n}$ :

$$
\varphi_{n}(z)=\Phi_{Q_{0}}^{n}(z) \tilde{s}\left(e^{-2 Q_{1}} ; z\right),
$$

where the normalization constant in (5) equals $C_{n}=\varphi_{n}^{-1}(\infty)$.

2.3. The first transformation $Y \rightarrow T$ : normalization of the matrix problem. First we recall that the goal of the first transformation is to make the solution holomorphic at infinity. To that end, consider a function $\varphi$ that solves the boundary value problem (5) with $W:=W_{n}$ (the index $n$ in $\varphi_{n}$, which denotes the dependence on $n$, has been omitted to simplify notation).

Using the diagonal matrices

$$
\Phi:=\operatorname{diag}\left\{\varphi^{-1}, \varphi\right\} \quad \text { and } \quad C:=\operatorname{diag}\left\{C_{n}^{-1}, C_{n}\right\},
$$

we define the matrix function

$$
T:=C Y \Phi=\left(\begin{array}{cc}
\frac{Q_{n}}{C_{n} \varphi} & \frac{\varphi}{C_{n}} R_{n} \\
\frac{C_{n} m \tilde{Q}_{n-1}}{\varphi} & C_{n} m \varphi \tilde{R}_{n-1}
\end{array}\right) .
$$

This function is the unique solution of the boundary value problem

$$
\begin{gathered}
T \in H_{2 \times 2}^{2}(\overline{\mathbb{C}} \backslash F), \\
T_{+}=T_{-} J \quad \text { on } \quad F, \\
T(z)=I+O\left(\frac{1}{z}\right), \quad z \rightarrow \infty,
\end{gathered}
$$

where, due to the identity

$$
T_{+}=C Y_{+} \Phi_{+}=C Y_{-} \mathbf{W} \Phi_{+}=C Y_{-} \Phi_{-} \Phi_{-}^{-1} \mathbf{W} \Phi_{+}=T_{-} \Phi_{-}^{-1} \mathbf{W} \Phi_{+},
$$

the jump matrix $J$ is given by

$$
J:=\Phi_{-}^{-1} \mathbf{W} \Phi_{+}=\left(\begin{array}{cc}
\frac{\varphi_{-}}{\varphi_{+}} & \varphi_{+} \varphi_{-} W_{n} \\
0 & \frac{\varphi_{+}}{\varphi_{-}}
\end{array}\right) \text {on } F .
$$

Note that on the support of the equilibrium measure, in view of the boundary conditions for the function $\varphi$, the jump matrix $J$ can be rewritten as

$$
J=\left(\begin{array}{cc}
\frac{\varphi_{-}}{\varphi_{+}} & \varphi_{+} \varphi_{-} W_{n} \\
0 & \frac{\varphi_{+}}{\varphi_{-}}
\end{array}\right)=\left(\begin{array}{cc}
\frac{\varphi_{-}}{\varphi_{+}} & 1 \\
0 & \frac{\varphi_{+}}{\varphi_{-}}
\end{array}\right) \text {on } \Delta .
$$

2.4. The second transformation $T \rightarrow S$ : factorization of the jump matrix and "opening the lens". Since the boundary values of the complex potential of the equilibrium measure $\lambda$,

$$
\mathcal{V}_{\lambda}(z)=-\int_{\Delta} \ln (z-t) d \lambda(t)=V_{\lambda}(z)-i \int_{\Delta} \arg (z-t) d \lambda(t)
$$

satisfy

$$
\mathcal{V}_{\lambda \pm}(z)=V_{\lambda}(z)-i \int_{a}^{z} \arg (z-t) d \lambda(t)+i \int_{b}^{z}[\arg (z-t) \pm \pi] d \lambda(t)
$$


on $\Delta$, we have that

$$
\frac{\Phi_{Q_{0} \mp}^{n}}{\Phi_{Q_{0} \pm}^{n}}=\exp \left\{-n\left(\mathcal{V}_{\lambda_{\mp}}-\mathcal{V}_{\lambda_{ \pm}}\right)\right\}=\exp \left\{2 \pi i n \ell_{ \pm}^{b}\right\}
$$

on $\Delta$, where

$$
\ell_{ \pm}^{b}:=\mp \int_{z}^{b} d \lambda(t), \quad z \in \Delta
$$

In view of the positivity of the measure $\lambda$ we have

$$
\operatorname{Im}\left(\ell_{ \pm}^{b}\right)=0 \text { on } \Delta .
$$

The fact that the external field $Q_{0}$ is holomorphic in $\Omega$ implies that the equilibrium measure is absolutely continuous and that its density $\lambda^{\prime}$ is holomorphic at all the interior points of the arc $\Delta$ and therefore it is holomorphic in a lens-shaped domain $d: \Delta \subset \bar{d} \subset \Omega$. Consequently, the function

$$
\ell^{b}(z)=\left\{\begin{array}{ll}
-\int_{x}^{b} \lambda^{\prime}(t) d t, & z \in d^{+}, \\
\int_{x}^{b} \lambda^{\prime}(t) d t, & z \in d^{-},
\end{array} \quad \overline{d^{+} \cup \Delta \cup d^{-}}=\bar{d},\right.
$$

being the primitive of a holomorphic function, is a holomorphic continuation of the functions $\ell_{+}^{b}$ and $\ell_{-}^{b}$ from the "banks" of the arc $\Delta$ to the simply connected domains $d^{+}$and $d^{-}$. Furthermore, $\operatorname{Im}(\ell)>0$ in $d^{+} \cup d^{-}$. Indeed, by the Cauchy-Riemann relations,

$$
\left.\frac{\partial \operatorname{Im}\left(\ell^{b}\right)}{\partial n}\right|_{x}=\left.\frac{\partial \operatorname{Re}\left(\ell^{b}\right)}{\partial \tau}\right|_{x}=\lambda^{\prime}(x)>0, \quad x \in \Delta .
$$

The jump matrix $J$ (see (22) ) can be neatly factorised on $\Delta$ :

$$
J=\left(\begin{array}{cc}
1 & 0 \\
\varphi_{+} / \varphi_{-} & 1
\end{array}\right)\left(\begin{array}{rr}
0 & 1 \\
-1 & 0
\end{array}\right)\left(\begin{array}{cc}
1 & 0 \\
\varphi_{-} / \varphi_{+} & 1
\end{array}\right) .
$$

We will denote the middle matrix, which does not depend on $n$, by $\mathcal{J}$ :

$$
\mathcal{J}:=\left(\begin{array}{rr}
0 & 1 \\
-1 & 0
\end{array}\right)
$$

Defining the matrix function

$$
D:=\left(\begin{array}{cc}
1 & 0 \\
1 /\left(W_{n} \varphi^{2}\right) & 1
\end{array}\right)
$$

in the domain $\Omega \backslash F$ and using the boundary condition of problem (5), we see that the factorization of the matrix $J$ on $\Delta$ has the form

$$
J=D_{-} \mathcal{J} D_{+} \quad \text { on } \quad \Delta .
$$

On $\Delta$, the boundary values of the left lower corner element of the matrix $D$ can be written as

$$
\frac{1}{W_{n} \varphi_{\mp}^{2}}=\frac{1}{e^{-2 n Q_{0}} \tilde{W} \tilde{s}_{ \pm}^{2} \Phi_{Q_{0} \pm}^{2 n}}=\left(\frac{\tilde{s}_{ \pm}}{\tilde{s}_{\mp}}\right)\left(\frac{\Phi_{Q_{0} \pm}}{\Phi_{Q_{0} \mp}}\right)^{n} \quad \text { on } \quad \Delta,
$$

where the first factor admits a bounded analytic continuation to $\Omega \backslash \Delta$, while the second factor can be transformed to the form (23). Furthermore, as we have already noted, an important consequence of the $S$-property of the $\operatorname{arc} \Delta$ is the fact that $\ell^{b}$ can be continued from both "banks" of $\Delta$ into the lens-shaped domains $d^{+}$and $d^{-}$, so that

$$
\operatorname{Im}\left(\ell^{b}\right)>0 \text { in } \quad d^{+} \cup d^{-} .
$$




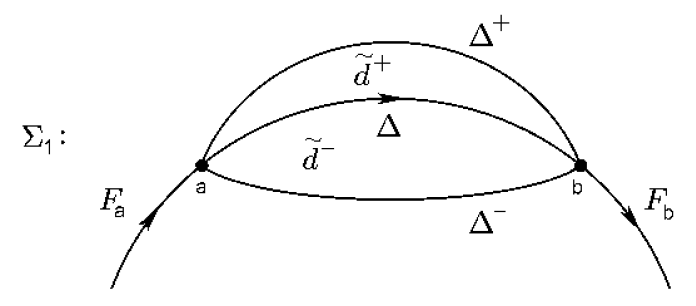

Figure 1. The contour $\Sigma_{1}$

Thus, on $\Delta$ the jump matrix $J$ admits the factorization (29), where the middle matrix $\mathcal{J}$ is independent of $n$, while the analytic continuation of the left and right matrices $D_{+}$ and $D_{-}$in the domains $d^{+}$and $d^{-}$, respectively, due to the identity

$$
\exp \left\{2 \pi i n \ell^{b}\right\}=\exp \left\{2 \pi i n\left(\operatorname{Re}\left(\ell^{b}\right)+i \operatorname{Im}\left(\ell^{b}\right)\right)\right\}=\exp \left\{-2 \pi n \operatorname{Im}\left(\ell^{b}\right)\right\} \exp \left\{2 \pi i n \operatorname{Re}\left(\ell^{b}\right)\right\},
$$

has the property that

$$
D_{\alpha} \rightrightarrows I \quad \text { as } \quad n \rightarrow \infty \quad \text { on } \quad K \Subset d^{\alpha}, \quad \alpha=+,-,
$$

and the rate is that of a geometric progression.

Let $\Delta^{+}$be an arc contained in $d^{+}$which connects the endpoints of the $\operatorname{arc} \Delta$. Similarly, $\Delta^{-}$is contained in $d^{-}$and connects the endpoints of $\Delta$. The $\operatorname{arcs} \Delta^{+}, \Delta^{-}$have the same orientation as $\Delta$, that is, from the point $a$ to the point $b$. Denote by $\tilde{d}^{+}$the domain lying between the $\operatorname{arcs} \Delta^{+}$and $\Delta$; similarly, $\tilde{d}^{-}$lies between the $\operatorname{arcs} \Delta^{-}$and $\Delta$ :

$$
\tilde{d}^{\alpha} \subset d^{\alpha}, \quad \Gamma\left(\tilde{d}^{\alpha}\right)=\Delta^{\alpha} \cup \Delta, \quad \alpha=+,-.
$$

Finally, denote by $F_{a}$ the part of the $\operatorname{arc} F$ that connects the initial point of the arc $F$ with the initial point of $\Delta$, and similarly, let $F_{b}$ denote the part of $F$ that connects the endpoint of $\Delta$ with the endpoint of $F$ :

$$
F=F_{a} \cup \Delta \cup F_{b} .
$$

Thus we pass from the problem (20) with boundary conditions on the arc $F$ to a problem with boundary conditions on the contour $\Sigma_{1}$ (see Figure 1):

$$
\Sigma_{1}:=F \cup \Delta^{+} \cup \Delta^{-}=F_{a} \cup \Delta \cup \Delta^{+} \cup \Delta^{-} \cup F_{b} .
$$

To that end, let us define the following piecewise-analytic matrix-valued function $S$ :

$$
S:=\left\{\begin{array}{lll}
T & \text { in } & \overline{\mathbb{C}} \backslash \overline{\left(\tilde{d}^{+} \cup \tilde{d}^{-}\right)}, \\
T D^{-1} & \text { in } & \tilde{d}^{+}, \\
T D & \text { in } & \tilde{d}^{-} .
\end{array}\right.
$$

By definition, for the boundary values of $S$ on $\Delta^{+}$and $\Delta^{-}$, we have

$$
\begin{array}{ll}
\text { on } \Delta^{+}: & S_{+}=T=T D^{-1} D=S_{-} D, \\
\text { on } \Delta^{-}: & S_{+}=T D=S_{-} D,
\end{array}
$$

and by taking (29) into account,

$$
\text { on } \Delta: \quad S_{+}=T_{+} D_{+}^{-1}=T_{-} J D_{+}^{-1}=T_{-} D_{-} \mathcal{J} D_{+} D_{+}^{-1}=S_{-} \mathcal{J} .
$$


Therefore the function $S$ is the unique solution of the following boundary value problem:

$$
\begin{gathered}
S \in H_{2 \times 2}^{2}\left(\overline{\mathbb{C}} \backslash \Sigma_{1}\right), \\
S_{+}=S_{-}\left\{\begin{array}{ccc}
D & \text { on } & \Delta^{+} \cup \Delta^{-}, \\
\mathcal{J} & \text { on } & \Delta, \\
J & \text { on } & F_{a} \cup F_{b},
\end{array}\right. \\
S(z)=I+O\left(\frac{1}{z}\right), \quad z \rightarrow \infty,
\end{gathered}
$$

where the jump functions are defined in (28), (27), and (21).

2.5. Analysis of the boundary value problem for $S$. The boundary value problem (33) has many advantages over the equivalent boundary value problem (20), such as:

a) the jump matrix $J$ is independent of $n$ on the segment $\Delta$ of the boundary contour $\Sigma_{1}$

b) the jump matrix tends to the identity matrix at "interior" points of the $\operatorname{arcs} \Delta^{+}$, $\Delta^{-}, F_{a}$ and $F_{b}$.

Condition a) is obvious. We will explain b). Let us fix some neighbourhoods of the endpoints of the $\operatorname{arc} \Delta$ :

$$
O_{a} \text { is a neighbourhood of } a, O_{b} \text { is a neighbourhood of } b .
$$

Next, denote the ends of the arcs of the contour $\Sigma_{1}$ adjacent to the endpoints of $\Delta$ by

$$
\Delta_{\alpha}^{ \pm}:=\Delta^{ \pm} \cap O_{\alpha}, \quad F_{\alpha}^{\varepsilon}:=F_{\alpha} \cap O_{\alpha}, \quad \alpha=a, b .
$$

Then by (30),

$$
D \rightrightarrows I \quad \text { as } \quad n \rightarrow \infty \quad \text { on } \quad \Delta^{\alpha} \backslash\left(\Delta_{a}^{\alpha} \cup \Delta_{b}^{\alpha}\right), \quad \alpha=+,-.
$$

Next let us note that, by the definition of the function $\varphi$, the jump matrix $J$ (see (21)) becomes

$$
J=\left(\begin{array}{cc}
1 & \varphi^{2} W_{n} \\
0 & 1
\end{array}\right)=\left(\begin{array}{cc}
1 & \tilde{W} \tilde{s}^{2}(\tilde{W} ; z) \exp \left\{2 n\left(\Gamma-\mathcal{V}_{\lambda}-Q_{0}\right)\right\} \\
0 & 1
\end{array}\right)
$$

on $F$ outside $\Delta$. By assumption, the function $\tilde{W}$ is bounded in $\Omega$ and therefore

$$
\tilde{W}(z) \tilde{s}^{2}(\tilde{W} ; z) \leq C \quad \text { on } \quad K \Subset \Omega .
$$

Since the measure $d \lambda$ is an equilibrium measure, we have

$$
\mathfrak{R}\left(\Gamma-\mathcal{V}_{\lambda}-Q_{0}\right)=\gamma-V_{\lambda}-\mathfrak{R}\left(Q_{0}\right) \leq 0
$$

on $F$ outside $\Delta$. Hence

$$
J \rightrightarrows I \text { as } n \rightarrow \infty \text { on } F_{\lambda} \backslash F_{\lambda}^{\varepsilon}, \lambda=a, b .
$$

Furthermore, the uniform convergence in (34) and in (35) is at a rate of a geometric progression.

Thus, in $\overline{\mathbb{C}} \backslash O_{\alpha}, \alpha=a, b$, we can expect that $S(z) \rightarrow N(z)$, where $N(z)$ is the solution of the following boundary value problem:

$$
\begin{gathered}
N \in H_{2 \times 2}^{2}(\overline{\mathbb{C}} \backslash \Delta), \\
N_{+}=N_{-} \mathcal{J} \text { on } \quad \Delta, \\
N(z)=I+O\left(\frac{1}{z}\right), \quad z \rightarrow \infty .
\end{gathered}
$$


Since the jump matrix is independent of $n$ and admits a convenient diagonalization, the solution of this problem can be found in an explicit form (see [22]):

$$
N=\left(\begin{array}{cc}
\frac{\beta+\beta^{-1}}{2} & \frac{\beta-\beta^{-1}}{2 i} \\
-\frac{\beta-\beta^{-1}}{2 i} & \frac{\beta+\beta^{-1}}{2}
\end{array}\right) .
$$

Here $\beta=\left(\frac{z-b}{z-a}\right)^{1 / 4}$.

But there are difficulties in proving rigorously that indeed $S \rightarrow N$ as $n \rightarrow \infty$. This can be seen by considering the "error"

$$
M:=S(N)^{-1} \text {. }
$$

Let us denote the joint jump matrix for $S$ by $\nu_{s}$. The matrix function $M$ then satisfies the following Riemann-Hilbert problem:

$$
\begin{gathered}
M_{+}=M_{-}\left(N_{-} \nu_{s} \mathcal{J}^{-1}\left(N_{-}\right)^{-1}\right) \quad \text { on the union of the contours } \Sigma_{1}, \\
M(z)=I+O\left(l \frac{1}{|z|}\right) \quad \text { as } \quad|z| \rightarrow \infty .
\end{gathered}
$$

To prove that the solution $M$ of (38) converges to the identity matrix, that is, to show that $S \approx N$, it is necessary to show that the jump matrix of $M$ converges to the identity matrix both in the $L_{2}$ and in the $L_{\infty}$-norm (see [3, 20]). However, since $N$ is singular at $a$ and $b$, the jump matrix for $M$ is unbounded. To circumvent this difficulty, we have to construct an exact solution of the problem (33) in a "small" neighbourhood of each of the points $a$ and $b$ that coincides with $N$ on the boundary of these neighbourhoods up to order $O\left(1 / n^{k}\right)$, where $k>0$ is the chosen order of truncation of the asymptotic series.

2.6. The formulation of the auxiliary boundary value problem in a neighbourhood of each endpoint of the support $\Delta$. In a neighbourhood of an endpoint of the support of the equilibrium measure, jump matrices to do not converge to identity matrices as $n \rightarrow \infty$. Consider the following boundary value problem:

$$
\begin{gathered}
U_{a} \in H_{2 \times 2}^{2}\left(O_{0} \backslash\left(\Delta_{a} \cup \Delta_{a}^{+} \cup \Delta_{a}^{-} \cup F_{a}^{\varepsilon}\right)\right), \\
U_{a+}=U_{a-}\left\{\begin{array}{lll}
D & \text { on } & \Delta_{a}^{+} \cup \Delta_{a}^{-}, \\
\mathcal{J} & \text { on } & \Delta_{a}, \\
J & \text { on } & F_{a}^{\varepsilon},
\end{array}\right. \\
U_{a}(z)=(I(z)+o(1)) N \text { as } z \in O_{a}, \quad n \rightarrow \infty .
\end{gathered}
$$

Exactly the same problem with $a$ replaced by $b$ in the subscript, is posed in a neighbourhood $O_{b}$ of the other endpoint $b$ for the function $U_{b}$.

Finding the solution of (39) is the main technical feature of the approach we are following here (see [18, 22]). The analysis of this boundary value problem depends on two properties. First of all, does the endpoint of $\Delta$ coincide with the endpoint of the arc $F$, the support of the orthogonality measure, or is the point $b$ an interior point of $F$ ? That is, we need to know whether the set $F_{b}^{\varepsilon}$ is empty or not. We thus have two cases:

$$
(A):=\left\{F_{b}^{\varepsilon} \neq \varnothing\right\}, \quad(B):=\left\{F_{b}^{\varepsilon}=\varnothing\right\} .
$$

Secondly, the analysis of (39) depends on the behaviour of the derivative $\lambda^{\prime}$ of the equilibrium measure in a neighbourhood of an endpoint of $\Delta$.

In this work, we shall consider the following regular cases:

$$
\begin{array}{llll}
\left\{F_{b}^{\varepsilon} \neq \varnothing\right\}, & \lambda^{\prime}(z)=(z-b)^{1 / 2} h(z), & h \in H\left(O_{b}\right), & h(b) \neq 0 . \\
\left\{F_{a}^{\varepsilon} \neq \varnothing\right\}, & \lambda^{\prime}(z)=(z-a)^{1 / 2} h(z), & h \in H\left(O_{a}\right), & h(a) \neq 0 .
\end{array}
$$




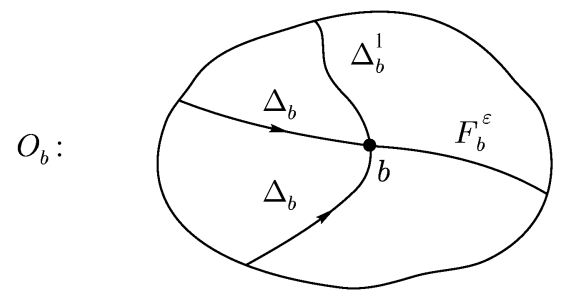

Figure 2. The contour $\Sigma_{2}$

2.7. Solving the boundary value problem in a neighbourhood of an endpoint using Airy functions. Let us show in detail how to construct the solution of the following boundary value problem:

$$
\begin{gathered}
U_{b} \in H_{2 \times 2}^{2}\left(O_{b} \backslash \Sigma_{b}\right), \\
U_{b+}=U_{b-}\left\{\begin{array}{ccc}
D & \text { on } \Delta_{b}^{+} \cup \Delta_{b}^{-}, \\
\mathcal{J} & \text { on } \Delta_{b}, \\
J & \text { on } F_{b}^{\varepsilon} \neq \varnothing,
\end{array}\right. \\
U_{b}(z)=(I+o(1)) N \text { on } \partial O_{b} \text { as } n \rightarrow \infty,
\end{gathered}
$$

where (see Figure 2)

$$
\Sigma_{b}:=\Delta_{b} \cup \Delta_{b}^{-} \cup \Delta_{b}^{+} \cup F_{b}^{\varepsilon} .
$$

In (43) the jump matrices are:

$$
\begin{aligned}
D & :=\left(\begin{array}{cc}
1 & 0 \\
1 /\left(W_{n} \varphi^{2}\right) & 1
\end{array}\right) \quad \text { in } \Omega \backslash F, \\
\mathcal{J} & :=\left(\begin{array}{cc}
0 & 1 \\
-1 & 0
\end{array}\right) \text { on } \Delta, \\
J & :=\left(\begin{array}{cc}
1 & W_{n} \varphi^{2} \\
0 & 1
\end{array}\right) \quad \text { on } F_{b}^{\varepsilon},
\end{aligned}
$$

and the matrix $N$ given by (37) does not depend on $n$.

2.7.1. Transformation of the latter problem into a problem in which the jump is independent of $n$. Denote by $A$ the diagonal matrix

$$
A:=\left(\begin{array}{cc}
\varphi W_{n}^{1 / 2} & 0 \\
0 & \left(\varphi W_{n}^{1 / 2}\right)^{-1}
\end{array}\right) .
$$

Note that the jump matrices (44) allow the following decompositions:

$$
\begin{aligned}
D & :=A\left(\begin{array}{ll}
1 & 0 \\
1 & 1
\end{array}\right) A^{-1} \quad \text { in } \quad \Omega \backslash F, \\
\mathcal{J} & :=A_{-}\left(\begin{array}{cc}
0 & \left(\varphi_{+} \varphi_{-} W_{n} \omega\right)^{-1} \\
\varphi_{+} \varphi_{-} W_{n} \omega & 0
\end{array}\right) A_{+}^{-1} \\
& =A_{-}\left(\begin{array}{cc}
0 & 1 \\
-1 & 0
\end{array}\right) A_{+}^{-1} \text { on } \Delta, \\
J & :=A\left(\begin{array}{ll}
1 & 1 \\
0 & 1
\end{array}\right) A^{-1} \quad \text { on } \quad F_{b}^{\varepsilon},
\end{aligned}
$$

where $\omega:=\frac{1}{i} w_{\Delta}=\frac{1}{i} \sqrt{(z-a)(z-b)}$. 


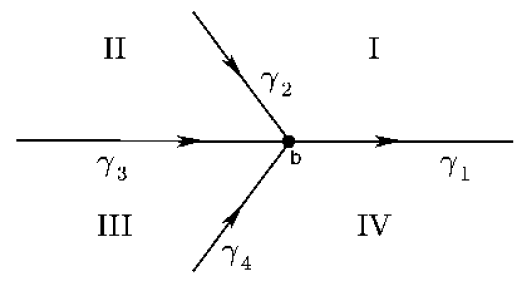

Figure 3. The auxiliary contour $\gamma_{0}$

We define the matrix function

$$
V_{b}=U_{b} A \text {. }
$$

Then, using (46), the boundary value problem (43) transforms into the following problem for $V_{b}$ :

$$
\begin{gathered}
V_{b} \in H_{2 \times 2}^{2}\left(O_{b} \backslash \Sigma_{b}\right), \\
V_{b+}=V_{b-}\left\{\begin{array}{l}
\left(\begin{array}{rr}
1 & 0 \\
1 & 1
\end{array}\right) \quad \text { on } \Delta_{b}^{+} \cup \Delta_{b}^{-}, \\
\left(\begin{array}{rr}
0 & 1 \\
-1 & 0
\end{array}\right) \quad \text { on } \Delta_{b}, \\
\left(\begin{array}{rr}
1 & 1 \\
0 & 1
\end{array}\right) \quad \text { on } F_{b}^{\varepsilon} \neq \varnothing, \\
V_{b}(z)=(I+o(1)) N A \text { on } \partial O_{b} \quad \text { as } n \rightarrow \infty .
\end{array}\right.
\end{gathered}
$$

2.7.2. The auxiliary problem for Airy functions. Let us introduce the auxiliary contour

$$
\gamma_{0}:=\gamma_{1} \cup \gamma_{2} \cup \gamma_{3} \cup \gamma_{4},
$$

which, as is shown in Figure 3, divides the complex plane into the four domains denoted by I, II, III, IV. We shall assume that the angle between $\gamma_{2}$ and $\gamma_{3}$ is in $(\pi / 3, \pi)$.

We use the notation

$$
\begin{aligned}
A^{u}(\zeta) & :=\left(\begin{array}{cc}
\operatorname{Ai}(\zeta) & \operatorname{Ai}\left(\varepsilon_{3}^{2} \zeta\right) \\
\operatorname{Ai}^{\prime}(\zeta) & \varepsilon_{3}^{2} \operatorname{Ai}^{\prime}\left(\varepsilon_{3}^{2} \zeta\right)
\end{array}\right), \\
A^{l} & :=\left(\begin{array}{cc}
\operatorname{Ai}(\zeta) & -\varepsilon_{3}^{2} \operatorname{Ai}\left(\varepsilon_{3} \zeta\right) \\
\operatorname{Ai}^{\prime}(\zeta) & -\operatorname{Ai}^{\prime}\left(\varepsilon_{3} \zeta\right)
\end{array}\right),
\end{aligned}
$$

where $\mathrm{Ai}$ is the Airy function and $\varepsilon_{3}=e^{2 \pi i / 3}$ is a root of unity. We remind the reader that Airy functions solve the differential equation (see [29])

$$
\operatorname{Ai}^{\prime \prime}(\zeta)=\zeta \operatorname{Ai}(\zeta), \quad \operatorname{Ai} \in H(\mathbb{C}) .
$$

Now define the piecewise-analytic function

$$
\Psi:=\left\{\begin{array}{lll}
A^{u} e^{\frac{-i \pi}{6} \sigma_{3}} & \text { in } & \text { I, } \\
A^{u} e^{\frac{-i \pi}{6} \sigma_{3}}\left(\begin{array}{rr}
1 & 0 \\
-1 & 1
\end{array}\right) & \text { in } & \text { II, } \\
A^{l} e^{\frac{-i \pi}{6} \sigma_{3}}\left(\begin{array}{ll}
1 & 0 \\
1 & 1
\end{array}\right) & \text { in } & \text { III, } \\
A^{l} e^{\frac{-i \pi}{6} \sigma_{3}} & \text { in } & \text { IV },
\end{array}\right.
$$


where $\sigma_{3}$ is the Pauli matrix

$$
\sigma_{3}:=\left(\begin{array}{cc}
1 & 0 \\
0 & 1
\end{array}\right)
$$

We will use the notation

$$
\tilde{\sigma}=e^{\frac{-i \pi}{6} \sigma_{3}} .
$$

It is known (see 22]) that the function $\Psi$ solves the following boundary value problem:

$$
\begin{gathered}
\Psi \in H\left(\mathbb{C} \backslash \gamma_{0}\right), \quad \exists \Psi_{ \pm} \in \mathbb{C}\left(\gamma_{0}\right), \\
\Psi_{+}:=\Psi_{-} \begin{cases}\left(\begin{array}{ll}
1 & 0 \\
1 & 1
\end{array}\right) & \text { on } \gamma_{2} \cup \gamma_{4}, \\
\left(\begin{array}{rr}
0 & 1 \\
-1 & 0
\end{array}\right) & \text { on } \gamma_{3}, \\
\left(\begin{array}{ll}
1 & 1 \\
0 & 1
\end{array}\right) & \text { on } \gamma_{1} .\end{cases}
\end{gathered}
$$

2.7.3. The choice of the arcs $\Delta_{b}^{+}, \Delta_{b}^{-}, F_{b}^{\varepsilon}$ and of the boundary $\partial O_{b}$. We now assume that we are given a conformal mapping $f: O_{b} \rightarrow f\left(O_{b}\right) \in \mathbb{C}$ such that it satisfies $f(b)=0$ and maps the contour $\Sigma_{b} \cap O_{b}$ into $\gamma_{0} \cap f\left(O_{b}\right)$. Then clearly the function $\Psi(f(z))$ will satisfy the first two conditions of the problem (48). We note that in fact, in the contour $\Sigma_{b}$, it is only the arc $\Delta_{b}$ (a part of the arc of the support of the equilibrium measure) that is fixed, while we can choose the placement of the arcs $\Delta_{b}^{+}$and $\Delta_{b}^{-}$, as well as of the boundary $\partial O_{b}$. Similarly, the arc $F_{b}^{\varepsilon}$ can be chosen as required, since due to the analyticity of the weight function, the contour of integration can be deformed. Finally, we note that the jump relation for $\Psi(f(z))$ will not change if we left-multiply this function by any analytic matrix function. This gives us an additional degree of freedom to satisfy the third condition of (48).

Summarizing, we observe that for any conformal map $f: O_{b} \rightarrow f\left(O_{b}\right)$ that satisfies $f\left(\Sigma_{b} \cap O_{b}\right)=\gamma_{0} \cap f\left(O_{b}\right)$ (orientation-preserving) and for any analytic matrix function $E: O_{b} \rightarrow \mathbb{C}^{2 \times 2}$, the matrix function

$$
E(z) \Psi(f(z))
$$

satisfies the first two conditions of the problem (48).

We recall the definition of the function $\ell^{b}(24)$ :

$$
\ell_{ \pm}^{b}:=\mp \int_{z}^{b} d \lambda(t), \quad z \in \Delta .
$$

As we have already shown, it admits a holomorphic continuation to the domain $O_{b} \backslash \Delta_{b}$.

Next, it will be convenient to consider the function $\ell^{b}$ multiplied by $i \pi$,

$$
\tilde{\ell^{b}}:=i \pi \ell^{b}, \quad \tilde{\ell}^{b}{ }_{ \pm}=\mp i \pi \int_{x}^{b} d \lambda=\mp i \pi \int_{x}^{b}(z-b)^{1 / 2} h(z) d z \quad \text { on } \quad \Delta_{b} .
$$

Integrating, we have

$$
\tilde{\ell^{b}}(z)=\frac{2}{3}(z-b)^{3 / 2} M(z), \quad \text { where } \quad M(z) \in H\left(O_{b}\right), \quad M(b) \neq 0 .
$$

Therefore the function

$$
\zeta(z):=\left(\frac{3}{2} \tilde{\ell}^{b}(z)\right)^{2 / 3}=(z-b) M^{2 / 3}(z)
$$

is a conformal mapping of the domain $O_{b}$ into some neighbourhood of the point 0 . From this it follows that $\zeta(z)$ takes purely complex values for $z \in \mathbb{C} \backslash \Delta$. 

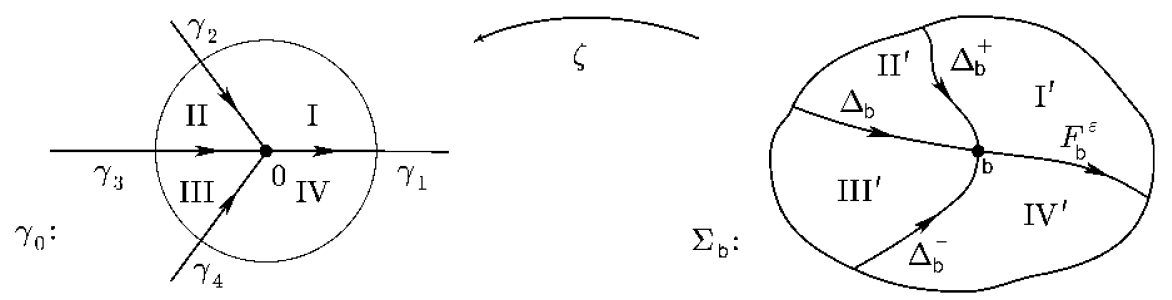

Figure 4 . The action of the function $\zeta$

Furthermore, this mapping takes the arc $\Delta_{b}$ into $\gamma_{3}$. Let us verify this (following [22]). We first consider the contour $\Sigma_{b}^{\prime}$, conformally identical to the contour $\Sigma_{b}$, such that $\Delta_{b^{\prime}}$ $\in \mathbb{R}$ corresponds to the interval $\Delta_{b}, \Delta_{b^{\prime}}^{+}$corresponds to $\Delta_{b}^{+}, \Delta_{b^{\prime}}^{-}$corresponds to $\Delta_{b}^{-}$, and $F_{b^{\prime}}^{\varepsilon} \in \mathbb{R}$ corresponds to $F_{b}^{\varepsilon}$. Next, suppose there is a mapping which can be represented in a neighbourhood of $b^{\prime}$ as

$$
\zeta_{0}(w)=\left(w-b^{\prime}\right) M_{0}^{2 / 3}(w), \quad M_{0}\left(b^{\prime}\right) \neq 0, \quad M_{0}(w) \in H\left(O_{b^{\prime}}\right) .
$$

In the expression above, the branch of the root is chosen so that $M_{0}^{2 / 3}\left(b^{\prime}\right)>0$. Then it is clear that $\zeta_{0}$ is analytic in a neighbourhood of $b^{\prime}, \zeta_{0}^{\prime}(a) \neq 0$. Therefore $\zeta_{0}$ is a one-to-one mapping of a neighbourhood of $b^{\prime}$ into a neighbourhood of zero. It is also clear that $\zeta_{0}$ maps real points into real points and imaginary points into imaginary ones. From connectedness and continuity we conclude that $\zeta_{0}\left(\mathbb{C}_{+} \cap O_{b^{\prime}}\right)$ is entirely contained either in the upper half-plane $\mathbb{C}_{+}$or in $\mathbb{C}_{-}$(and similarly for $\zeta_{0}\left(\mathbb{C}_{-} \cap O_{b^{\prime}}\right)$ ). Since for sufficiently small $\varepsilon>0$

$$
\begin{aligned}
\zeta_{0}\left(b^{\prime}+i \varepsilon\right) & =i \varepsilon\left(M_{0}(a)+i \varepsilon M_{0}^{\prime}(a)+\cdots\right)^{2 / 3}=i \varepsilon\left(M_{0}(a)\right)^{2 / 3}(1+O(\varepsilon)) \\
& =i \varepsilon M_{0}(a)^{2 / 3}+O\left(\varepsilon^{2}\right),
\end{aligned}
$$

we have $\operatorname{Im}\left(\zeta_{0}\left(b^{\prime}+i \varepsilon\right)\right)>0$ for small positive $\varepsilon$. Therefore, $\zeta_{0}\left(\mathbb{C}_{+} \cap O_{b^{\prime}}\right) \in \mathbb{C}_{+}$and $\zeta_{0}\left(\mathbb{C}_{-} \cap O_{b^{\prime}}\right) \in \mathbb{C}_{-}$.

Let $\mu$ be a conformal mapping of $\Sigma_{b}^{\prime}$ into $\Sigma_{b}$. Then the composition $\mu \circ \zeta$ will satisfy all the conditions satisfied by $\zeta_{0}$ and therefore the mapping $\zeta$ takes the contour $\Sigma_{b}$ into the image of $\Sigma_{b^{\prime}}$ under the map $\zeta_{0}$.

2.7.4. "Sewing up" the boundary conditions on $\partial O_{b}$. Recall that we are looking for a solution of (48) in the form

$$
V_{b}(z)=E(z) \Psi\left(\tilde{c}_{n} \zeta(z)\right) .
$$

To satisfy the boundary conditions on the boundary $\partial O_{b}$ as $n \rightarrow \infty$, we need the asymptotics of $\Psi\left(\tilde{c}_{n} \zeta(z)\right)$ as $\tilde{c}_{n} \rightarrow \infty, n \rightarrow \infty$. Let us first consider the case $\zeta \in I$.

It is known that at infinity the Airy function admits the following expansion (see 29]):

$$
\begin{aligned}
& \operatorname{Ai}(\zeta) \sim \frac{1}{2 \sqrt{\pi}} \zeta^{-1 / 4} e^{-2 / 3 \zeta^{3 / 2}} \sum_{k=0}^{\infty}(-1)^{k} s_{k}\left(\frac{2}{3} \zeta^{3 / 2}\right)^{-k} \quad \text { for } \quad|\arg \zeta|<\pi, \\
& \operatorname{Ai}^{\prime}(\zeta) \sim-\frac{1}{2 \sqrt{\pi}} \zeta^{1 / 4} e^{-2 / 3 \zeta^{3 / 2}} \sum_{k=0}^{\infty}(-1)^{k} t_{k}\left(\frac{2}{3} \zeta^{3 / 2}\right)^{-k} \quad \text { for } \quad|\arg \zeta|<\pi,
\end{aligned}
$$

where

$$
s_{0}=t_{0}=1, \quad s_{k}=\frac{\Gamma(3 k+1 / 2)}{54^{k} k ! \Gamma(k+1 / 2)}, \quad t_{k}=-\frac{6 k+1}{6 k-1} s_{k} \quad \text { for } \quad k \geq 1,
$$

and $\Gamma$ denotes the gamma function. 
Setting

$$
\tilde{c}_{n}:=n^{2 / 3},
$$

for $\zeta \in \partial O_{b}$ we have

$$
\left|n^{2 / 3} \zeta\right|=\left|\varepsilon_{3}^{2} n^{2 / 3} \zeta\right|=n^{2 / 3} \epsilon \rightarrow \infty, \quad n \rightarrow \infty,
$$

where $\epsilon>0$ is the radius of $O_{b}$.

Substituting in (49) the asymptotic expansions (52), (53) (following [3]), we obtain

$$
\begin{aligned}
& \Psi\left(n^{2 / 3} \zeta\right)=\left(\begin{array}{cc}
\operatorname{Ai}\left(n^{2 / 3} \zeta\right) & \mathrm{Ai}\left(\varepsilon_{3}^{2} n^{2 / 3} \zeta\right) \\
\operatorname{Ai}^{\prime}\left(n^{2 / 3} \zeta\right) & \varepsilon_{3}^{2} \mathrm{Ai}^{\prime}\left(\varepsilon_{3}^{2} n^{2 / 3} \zeta\right)
\end{array}\right) \tilde{\sigma} \\
&=\frac{1}{2 \sqrt{\pi}}\left(\begin{array}{c}
\left(n^{2 / 3} \zeta\right)^{-1 / 4} e^{-2 / 3\left(n^{2 / 3} \zeta\right)^{3 / 2}}\left(\sum_{k=0}^{\infty}(-1)^{k} s_{k}\left(\frac{2}{3} n \zeta^{3 / 2}\right)^{-k}\right) \\
-\left(n^{2 / 3} \zeta\right)^{1 / 4} e^{-2 / 3\left(n^{2 / 3} \zeta\right)^{3 / 2}}\left(\sum_{k=0}^{\infty}(-1)^{k} t_{k}\left(\frac{2}{3} n \zeta^{3 / 2}\right)^{-k}\right) \\
\quad\left(\varepsilon_{3}^{2} n^{2 / 3} \zeta\right)^{-1 / 4} e^{-2 / 3\left(\varepsilon_{3}^{2} n^{2 / 3} \zeta\right)^{3 / 2}}\left(\sum_{k=0}^{\infty}(-1)^{k} s_{k}\left(\frac{2}{3}\left(\varepsilon_{3}^{2} n^{2 / 3} \zeta\right)^{3 / 2}\right)^{-k}\right) \\
-\varepsilon_{3}^{2}\left(\varepsilon_{3}^{2} n^{2 / 3} \zeta\right)^{1 / 4} e^{-2 / 3\left(\varepsilon_{3}^{2} n^{2 / 3} \zeta\right)^{3 / 2}}\left(\sum_{k=0}^{\infty}(-1)^{k} t_{k}\left(\frac{2}{3}\left(\varepsilon_{3}^{2} n^{2 / 3} \zeta\right)^{3 / 2}\right)^{-k}\right)
\end{array}\right) \tilde{\sigma} .
\end{aligned}
$$

Next, by the definition of $\varepsilon_{3}$ and $\zeta$, we have

$$
\left(\varepsilon_{3}^{2} \zeta\right)^{3 / 2}=|\zeta|^{3 / 2} \exp \left\{i\left(\arg \zeta-\frac{2 \pi}{3}\right) \frac{3}{2}\right\}=-(\zeta)^{3 / 2}=-\frac{3}{2} \tilde{\ell^{b}}
$$

Substituting this formula into the expression for $\Psi\left(n^{2 / 3} \zeta\right)$, the latter can be written as

$$
\begin{aligned}
& \Psi\left(n^{2 / 3} \zeta\right)=\frac{1}{2 \sqrt{\pi}}\left(\begin{array}{cc}
\left(n^{2 / 3} \zeta\right)^{-1 / 4} & 0 \\
0 & \left(n^{2 / 3} \zeta\right)^{1 / 4}
\end{array}\right) \\
& \times\left(\begin{array}{cc}
e^{-n \tilde{\ell^{b}}} \sum_{k=0}^{\infty}(-1)^{k} s_{k}\left(\frac{1}{\left(\tilde{\ell^{b}} n\right)^{k}}\right) & \left(\varepsilon_{3}^{2}\right)^{-1 / 4} e^{n \tilde{\ell^{b}}} \sum_{k=0}^{\infty} s_{k}\left(\frac{1}{\left(\tilde{\ell^{b}} n\right)^{k}}\right) \\
-e^{-n \tilde{\ell^{b}}} \sum_{k=0}^{\infty}(-1)^{k} t_{k}\left(\frac{1}{\left(\tilde{\tilde{\ell}^{b}} n\right)^{k}}\right) & -\varepsilon_{3}^{2}\left(\varepsilon_{3}^{2}\right)^{1 / 4} e^{n \tilde{\ell^{b}}} \sum_{k=0}^{\infty} t_{k}\left(\frac{1}{\left(\tilde{\tilde{\ell}^{b}} n\right)^{k}}\right)
\end{array}\right) \tilde{\sigma} \\
& =\frac{1}{2 \sqrt{\pi}}\left(\begin{array}{cc}
\left(n^{2 / 3} \zeta\right)^{-1 / 4} & 0 \\
0 & \left(n^{2 / 3} \zeta\right)^{1 / 4}
\end{array}\right) \sum_{k=0}^{\infty}\left(\begin{array}{cc}
(-1)^{k} s_{k} & \left(\varepsilon_{3}^{2}\right)^{-1 / 4} s_{k} \\
-(-1)^{k} t_{k} & -\varepsilon_{3}^{2}\left(\varepsilon_{3}^{2}\right)^{1 / 4} t_{k}
\end{array}\right) \\
& \times\left(\begin{array}{cc}
e^{-i \pi / 6} & 0 \\
0 & e^{i \pi / 6}
\end{array}\right)\left(\begin{array}{cc}
e^{-n \tilde{\ell^{b}}} & 0 \\
0 & e^{n \tilde{\ell^{b}}}
\end{array}\right)\left(\frac{1}{\left(\tilde{\ell^{b}} n\right)^{k}}\right) \\
& =\frac{e^{\pi i / 12}}{2 \sqrt{\pi}}\left(\begin{array}{cc}
\left(n^{2 / 3} \zeta\right)^{-1 / 4} & 0 \\
0 & \left(n^{2 / 3} \zeta\right)^{1 / 4}
\end{array}\right) \sum_{k=0}^{\infty}\left(\begin{array}{cc}
(-1)^{k} s_{k} & s_{k} \\
-(-1)^{k} t_{k} & t_{k}
\end{array}\right) \\
& \times\left(\begin{array}{cc}
e^{-i \pi / 4} & 0 \\
0 & e^{i \pi / 4}
\end{array}\right)\left(\begin{array}{cc}
e^{-n \tilde{\ell^{b}}} & 0 \\
0 & e^{n \tilde{\ell^{b}}}
\end{array}\right)\left(\frac{1}{\left(\tilde{\tilde{\ell}^{b}} n\right)^{k}}\right) \\
& =\frac{\left(n^{2 / 3} \zeta\right)^{-\sigma_{3} / 4}}{2 \sqrt{\pi}}\left(\begin{array}{cc}
e^{-i \pi / 6} & e^{i \pi / 3} \\
-e^{-i \pi / 6} & -e^{4 i \pi / 3}
\end{array}\right)\left(\begin{array}{cc}
e^{-n \tilde{\ell^{b}}} & 0 \\
0 & e^{n \tilde{\ell^{b}}}
\end{array}\right)\left(I+O\left(\frac{1}{n}\right)\right) \text {. }
\end{aligned}
$$


The asymptotic formulae (55) and (56) have been derived for $\zeta \in I^{\prime} \cap \partial O_{b}$ (see Figure 4) Repeating the same argument for the other segments of $\partial O_{b}$ leads to the same formulae. Thus, (55) and (56) are valid uniformly on $\partial O_{b}$.

The boundary condition on $\partial O_{b}$ in (48), which must be satisfied, has the form

$$
E(z) \Psi\left(n^{2 / 3} \zeta(z)\right)=\left(I+O\left(\frac{1}{n}\right)\right) N A \quad \text { uniformly in } \quad z \in \partial O_{b} \quad \text { as } \quad n \rightarrow \infty,
$$

or

$$
E(z) \Psi\left(n^{2 / 3} \zeta(z)\right) A^{-1}=N+O\left(\frac{1}{n}\right) \quad \text { uniformly in } \quad z \in \partial O_{b} \quad \text { as } \quad n \rightarrow \infty,
$$

where

$$
A:=\operatorname{diag}\left\{\varphi W_{n}^{1 / 2},\left(\varphi W_{n}^{1 / 2}\right)^{-1}\right\}=\operatorname{diag}\left\{e^{-n \tilde{\ell^{b}}}, e^{n \tilde{\ell^{b}}}\right\} \operatorname{diag}\left\{s, s^{-1}\right\}
$$

and we have used the notation

$$
s:=\tilde{s}(\tilde{W} ; z) \tilde{W}^{1 / 2} .
$$

Let us put $S_{0}=\operatorname{diag}\left\{s, s^{-1}\right\}$.

We define the matrix function $E$ by the condition

$$
\begin{aligned}
& E(z) \frac{\left(n^{2 / 3} \zeta\right)^{-\sigma_{3} / 4}}{2 \sqrt{\pi}}\left(\begin{array}{cc}
e^{-i \pi / 6} & e^{i \pi / 3} \\
-e^{-i \pi / 6} & -e^{4 i \pi / 3}
\end{array}\right) S_{0}^{-1} \\
& \quad=N=\frac{1}{2}\left(\begin{array}{cc}
1 & 1 \\
i & -i
\end{array}\right)\left(\frac{z-b}{z-a}\right)^{\sigma_{3} / 4}\left(\begin{array}{cc}
1 & -i \\
1 & i
\end{array}\right) .
\end{aligned}
$$

The following decomposition holds:

$$
\begin{aligned}
\left(\begin{array}{rc}
e^{-i \pi / 6} & e^{i \pi / 3} \\
-e^{-i \pi / 6} & -e^{4 i \pi / 3}
\end{array}\right) & =\left(\begin{array}{rr}
0 & 1 \\
-1 & 0
\end{array}\right)\left(\begin{array}{ll}
e^{-i \pi / 6} & e^{4 i \pi / 3} \\
e^{-i \pi / 6} & e^{i \pi / 3}
\end{array}\right) \\
& =e^{-i \pi / 6}\left(\begin{array}{rr}
0 & 1 \\
-1 & 0
\end{array}\right)\left(\begin{array}{rr}
1 & -i \\
1 & i
\end{array}\right)
\end{aligned}
$$

Then

$$
E(z)=2 \sqrt{\pi} N S_{0}\left(\begin{array}{rc}
e^{-i \pi / 6} & e^{i \pi / 3} \\
-e^{-i \pi / 6} & -e^{4 i \pi / 3}
\end{array}\right)^{-1}\left(n^{2 / 3} \zeta(z)\right)^{\sigma_{3} / 4}
$$

or, in a more expanded form,

$$
\begin{aligned}
E(z)= & \frac{1}{2}\left(\begin{array}{cc}
1 & 1 \\
i & -i
\end{array}\right)\left(\frac{z-b}{z-a}\right)^{\sigma_{3} / 4}\left(\begin{array}{rr}
1 & -i \\
1 & i
\end{array}\right)\left(\begin{array}{cc}
s & 0 \\
0 & s^{-1}
\end{array}\right)\left(\begin{array}{rr}
1 & -i \\
1 & i
\end{array}\right)^{-1} \\
& \times\left(\begin{array}{rr}
0 & -1 \\
1 & 0
\end{array}\right) e^{i \pi / 6} 2 \sqrt{\pi}\left(n^{2 / 3} \zeta\right)^{\sigma_{3} / 4} \\
= & e^{i \pi / 6} \frac{\sqrt{\pi}}{2 s}\left(\begin{array}{cc}
1 & 1 \\
i & -i
\end{array}\right)\left(\frac{z-b}{z-a}\right)^{\sigma_{3} / 4}\left(\begin{array}{cc}
s^{2}-1 & -\left(s^{2}+1\right) \\
s^{2}+1 & s^{2}-1
\end{array}\right)\left(n^{2 / 3} \zeta\right)^{\sigma_{3} / 4} .
\end{aligned}
$$

We note that for $S_{0}=I$, for instance when $\tilde{W}=1$, we have

$$
E(z)=e^{i \pi / 6} \sqrt{\pi}\left(\begin{array}{cc}
1 & 1 \\
i & -i
\end{array}\right)\left(\begin{array}{rr}
0 & -1 \\
1 & 0
\end{array}\right)\left(\frac{z-a}{z-b} n^{2 / 3} \zeta\right)^{\sigma_{3} / 4}
$$

It remains to check that $E(z)$ is holomorphic in $O_{b}$.

Proposition 1. E $(z)$ is holomorphic in $O_{b}$. 
Proof. First we prove that $E$ has no jumps on $\Delta$.

In a neighbourhood of the point $b$ the functions $\beta, \beta^{-1}$ and $\zeta$ behave as follows:

$$
\beta(z) \simeq(z-b)^{1 / 4}, \quad \beta^{-1}(z) \simeq 1 /(z-b)^{1 / 4}, \quad \zeta(z) \simeq(z-b) .
$$

Furthermore, multiplying the matrices on the right-hand side of (58), we obtain (60)

$$
\begin{aligned}
E(z)= & \text { const } \\
& \times\left(\begin{array}{cc}
\left(\frac{1}{\beta}\left(s+s^{-1}\right)+\beta\left(s-s^{-1}\right)\right) \zeta^{1 / 4} & \left(\frac{1}{\beta}\left(-s+s^{-1}\right)-\beta\left(s+s^{-1}\right)\right) \zeta^{-1 / 4} \\
\left(\frac{1}{\beta}\left(-s-s^{-1}\right)+\beta\left(s-s^{-1}\right)\right) i \zeta^{1 / 4} & -\left(\frac{1}{\beta}\left(-s+s^{-1}\right)+\beta\left(s+s^{-1}\right)\right) i \zeta^{-1 / 4}
\end{array}\right) \\
& \times\left(n^{2 / 3}\right) \sigma_{3} / 4 .
\end{aligned}
$$

It is sufficient to verify that the middle matrix does not change as we cross $\Delta$. To indicate the method we will check this for the element in the right upper corner of this matrix (taking into account that $s_{+}=1 / s_{-}$):

$$
\begin{aligned}
& \left(\frac{1}{\beta_{+}}\left(s_{+}+s_{+}^{-1}\right)+\beta_{+}\left(s_{+}-s_{+}^{-1}\right)\right) \zeta_{+}^{1 / 4} \rightarrow\left(\frac{1}{\beta_{-}}\left(s_{-}+s_{-}^{-1}\right)+\beta_{-}\left(s_{-}-s_{-}^{-1}\right)\right) \zeta_{-}^{1 / 4} \\
= & \left(\frac{1}{i \beta_{+}}\left(s_{+}^{-1}+s_{+}\right)+i \beta_{+}\left(s_{+}^{-1}-s_{+}\right)\right) i \zeta_{+}^{1 / 4}=\left(\frac{1}{\beta_{+}}\left(s_{+}+s_{+}^{-1}\right)+\beta_{+}\left(s_{+}-s_{+}^{-1}\right)\right) \zeta_{+}^{1 / 4} .
\end{aligned}
$$

The verification for the other elements of the matrix is similar.

Finally we show that the point $b$ is a removable singularity of $E$. From the boundary conditions on $\Delta$ it follows that

$$
s \simeq \frac{1}{s} \simeq(z-b)^{-1 / 2} \quad \text { in } \quad O_{b},
$$

so that the elements in the matrix (60) cannot have a singularity at $b$ of order higher than $O\left(1 /(z-b)^{1 / 2}\right)$. As we know, the function $E$ is analytic in a punctured neighbourhood. Therefore, the isolated singularity $b$ is removable. Hence $E$ is analytic also in $O_{b}$.

Thus, having defined $E$ by (58), we have "sewn up" the condition on the boundary $\partial O_{b}$ :

$$
E(z) \Psi\left(n^{2 / 3} \zeta(z)\right) A^{-1}=N+O\left(\frac{1}{n}\right) \quad \text { uniformly in } z \in \partial O_{b} \quad \text { as } \quad n \rightarrow \infty .
$$

2.7.5. The local boundary value problem at a, the other endpoint of the support. Let us solve the boundary value problem also at the other endpoint of the support:

$$
\begin{gathered}
U_{a} \in H_{2 \times 2}^{2}\left(O_{a} \backslash\left(\Delta_{a} \cup \Delta_{a}^{+} \cup \Delta_{a}^{-} \cup F_{a}^{\varepsilon}\right)\right), \\
U_{a+}=U_{a-} \begin{cases}D & \text { on } \Delta_{a}^{+} \cup \Delta_{a}^{-}, \\
\mathcal{J} & \text { on } \Delta_{a}, \\
J & \text { on } F_{a}^{\varepsilon},\end{cases} \\
U_{a}(z)=(I(z)+o(1)) N, \quad \text { as } z \in O_{a}, \quad n \rightarrow \infty .
\end{gathered}
$$

Let us define the functions

$$
\ell_{ \pm}^{a}(z)= \pm \int_{a}^{z} d \lambda= \pm \int_{a}^{z}(t-a)^{1 / 2} h(t) d t, \quad z \in \mathbb{C} \backslash \Delta, \quad \tilde{\ell}^{a}(z)=\pi i \ell^{a}(z) .
$$

Clearly, the following relations hold:

$$
\left\{\begin{array}{l}
\tilde{\ell}_{+}^{b}+\pi i=\tilde{\ell}_{+}^{a}, \\
\tilde{\ell}_{-}^{b}-\pi i=\tilde{\ell}_{-}^{a} .
\end{array}\right.
$$

The matrix $A$ can now be written in the form

$$
A:=\operatorname{diag}\left\{\varphi W_{n}^{1 / 2},\left(\varphi W_{n}^{1 / 2}\right)^{-1}\right\}=\operatorname{diag}\left\{e^{-n \tilde{\ell^{a}}}, e^{n \tilde{\ell^{a}}}\right\} \operatorname{diag}\left\{s, s^{-1}\right\} .
$$



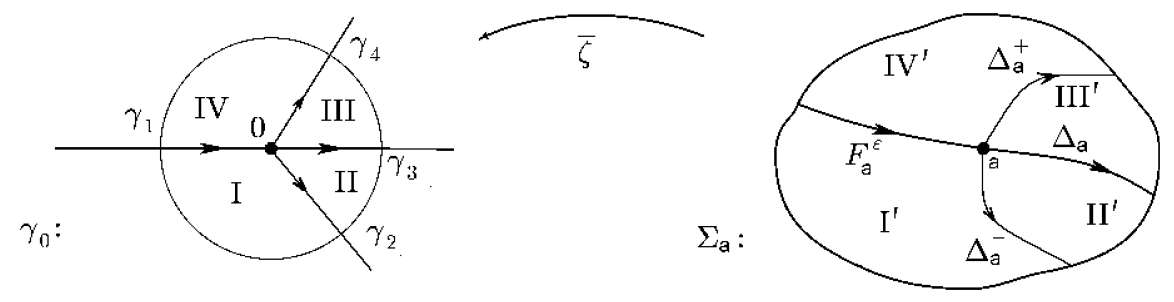

Figure 5 . The action of the mapping $\bar{\zeta}$

Next, consider the function

$$
\tilde{\zeta}(z):=-\left(\frac{3}{2} \tilde{\ell}^{a}(z)\right)^{2 / 3}=(z-a) M_{1}^{2 / 3}(z) .
$$

Arguing as in the case of the function (51), we can check that the function (61) realizes a conformal mapping of the domain $O_{a}$ into a neighbourhood of 0 , and that the contours are mapped as shown in Figure 5 .

Using the relations $\bar{\gamma}_{1}=-\gamma_{1}, \bar{\gamma}_{2}=-\gamma_{2}, \bar{\gamma}_{3}=-\gamma_{3}$ and $\bar{\gamma}_{4}=-\gamma_{4}$, it is not hard to show that the function $\bar{\Psi}(z)=\sigma_{3} \Psi(-z) \sigma_{3}$ (conjugation with the Pauli matrix takes into account the change in the direction of the rays) solves the problem

$$
\begin{gathered}
\bar{\Psi} \in H\left(\mathbb{C} \backslash \bar{\gamma}_{0}\right), \quad \exists \Psi_{ \pm} \in \mathbb{C}\left(\bar{\gamma}_{0}\right), \\
\bar{\Psi}_{+}:=\bar{\Psi}_{-}\left\{\begin{array}{ccc}
\left(\begin{array}{cc}
1 & 0 \\
1 & 1
\end{array}\right) & \text { on } & \bar{\gamma}_{2} \cup \bar{\gamma}_{4}, \\
\left(\begin{array}{rr}
0 & 1 \\
-1 & 0
\end{array}\right) & \text { on } & \bar{\gamma}_{3}, \\
\left(\begin{array}{cc}
1 & 1 \\
0 & 1
\end{array}\right) & \text { on } & \bar{\gamma}_{1} .
\end{array}\right.
\end{gathered}
$$

Using arguments similar to those for $\Psi\left(n^{2 / 3} \zeta\right)$, we see that the asymptotic expansion for $\bar{\Psi}\left(n^{2 / 3} \bar{\zeta}\right)$ takes the form

$$
\begin{aligned}
\bar{\Psi}\left(n^{2 / 3} \tilde{\zeta}\right)= & \sigma_{3} \Psi\left(-n^{2 / 3} \tilde{\zeta}\right) \sigma_{3}=\sigma_{3}\left(\begin{array}{cc}
\operatorname{Ai}\left(-n^{2 / 3} \tilde{\zeta}\right) & \operatorname{Ai}\left(-\varepsilon_{3}^{2} n^{2 / 3} \tilde{\zeta t a} a\right) \\
\operatorname{Ai}^{\prime}\left(-n^{2 / 3} \tilde{\zeta}\right) & \varepsilon_{3}^{2} \operatorname{Ai}^{\prime}\left(-\varepsilon_{3}^{2} n^{2 / 3} \tilde{\zeta}\right)
\end{array}\right) \tilde{\sigma} \sigma_{3} \\
= & \sigma_{3} \frac{e^{\pi i / 12}}{2 \sqrt{\pi}}\left(\begin{array}{cc}
\left(-n^{2 / 3} \tilde{\zeta}\right)^{-1 / 4} & 0 \\
0 & \left(-n^{2 / 3} \tilde{\zeta}\right)^{1 / 4}
\end{array}\right) \sum_{k=0}^{\infty}\left(\begin{array}{cc}
(-1)^{k} s_{k} & s_{k} \\
-(-1)^{k} t_{k} & t_{k}
\end{array}\right) \\
& \times\left(\begin{array}{cc}
e^{-i \pi / 4} & 0 \\
0 & e^{i \pi / 4}
\end{array}\right)\left(\begin{array}{cc}
e^{-n \tilde{\ell^{a}}} & 0 \\
0 & e^{n \tilde{\ell^{a}}}
\end{array}\right)\left(\begin{array}{cc}
1 \\
\left(\tilde{\ell^{a}} n\right)^{k}
\end{array}\right) \sigma_{3} \\
= & \sigma_{3} \frac{\left(-n^{2 / 3} \tilde{\zeta}\right)^{-\sigma_{3} / 4}}{2 \sqrt{\pi}}\left(\begin{array}{cc}
e^{-i \pi / 6} & e^{i \pi / 3} \\
-e^{-i \pi / 6} & -e^{4 i \pi / 3}
\end{array}\right)\left(\begin{array}{cc}
e^{-n \tilde{\ell}_{a}} & 0 \\
0 & e^{n \tilde{\ell^{a}}}
\end{array}\right)\left(I+O\left(\frac{1}{n}\right)\right) \sigma_{3} .
\end{aligned}
$$

Defining the function $\bar{E}$ by

$$
\begin{gathered}
\bar{E}(z) \sigma_{3} \frac{\left(-n^{2 / 3} \tilde{\zeta}(z)\right)^{-\frac{\sigma_{3}}{4}}}{2 \sqrt{\pi}}\left(\begin{array}{cc}
e^{-i \pi / 6} & e^{i \pi / 3} \\
-e^{-i \pi / 6} & -e^{4 i \pi / 3}
\end{array}\right) S_{0}^{-1} \sigma_{3} \\
=N=\frac{1}{2}\left(\begin{array}{cc}
1 & 1 \\
i & -i
\end{array}\right)\left(\frac{z-b}{z-a}\right)^{\sigma_{3} / 4}\left(\begin{array}{cc}
1 & -i \\
1 & i
\end{array}\right),
\end{gathered}
$$


we obtain

$$
\bar{E}(z)=2 \sqrt{\pi} N S_{0} \sigma_{3}^{-1}\left(\begin{array}{cc}
e^{-i \pi / 6} & e^{i \pi / 3} \\
-e^{-i \pi / 6} & -e^{4 i \pi / 3}
\end{array}\right)^{-1} \sigma_{3}^{-1}\left(-n^{2 / 3} \tilde{\zeta}(z)\right)^{\sigma_{3} / 4}
$$

For $S_{0}=I$,

$$
\bar{E}(z)=e^{i \pi / 6} \sqrt{\pi}\left(\begin{array}{cc}
1 & 1 \\
i & -i
\end{array}\right)\left(-\frac{z-b}{z-a} n^{2 / 3} \tilde{\zeta}(z)\right)^{\sigma_{3} / 4} .
$$

2.8. The final transformation, $S \rightarrow R$. We are now ready to effect the final transformation. We introduce the matrix function

$$
S_{\mathrm{par}}= \begin{cases}U_{a} & \text { on } O_{a}, \\ U_{b} & \text { on } O_{b}, \\ N & \text { elsewhere }\end{cases}
$$

Using the formulae derived in the previous section, we write down the explicit expressions for $U_{a}, U_{b}$ :

$$
\begin{aligned}
U_{b}:= & V_{b} A^{-1}=E(z) \Psi\left(\tilde{c}_{n} \zeta(z)\right) A^{-1} \\
= & e^{\pi i / 12} N S_{0}\left(\begin{array}{rr}
e^{-i \pi / 6} & e^{i \pi / 3} \\
-e^{-i \pi / 6} & -e^{4 i \pi / 3}
\end{array}\right) \\
& \times \sum_{k=0}^{\infty}\left(\begin{array}{rr}
(-1)^{k} s_{k} & s_{k} \\
-(-1)^{k} t_{k} & t_{k}
\end{array}\right)\left(\begin{array}{cc}
e^{-i \pi / 4} & 0 \\
0 & e^{i \pi / 4}
\end{array}\right) S_{0}^{-1}\left(\frac{1}{\left(\tilde{\ell}^{b} n\right)^{k}}\right), \\
U_{a}:= & V_{a} A^{-1}=\bar{E}(z) \bar{\Psi}\left(\tilde{c}_{n} \tilde{\zeta}(z)\right) A^{-1} \\
= & e^{\pi i / 12} N S_{0} \sigma_{3}\left(\begin{array}{cc}
e^{-i \pi / 6} & e^{i \pi / 3} \\
-e^{-i \pi / 6} & -e^{4 i \pi / 3}
\end{array}\right)^{-1} \\
& \times \sum_{k=0}^{\infty}\left(\begin{array}{rr}
(-1)^{k} s_{k} & s_{k} \\
-(-1)^{k} t_{k} & t_{k}
\end{array}\right) \sigma_{3}\left(\begin{array}{cc}
e^{-i \pi / 4} & 0 \\
0 & e^{i \pi / 4}
\end{array}\right) S_{0}^{-1}\left(\frac{1}{\left(\tilde{\tilde{\ell}^{a}} n\right)^{k}}\right) .
\end{aligned}
$$

Adding up the above transformations, we obtain (as in [3])

Proposition 2. Suppose

$$
R(z) \equiv S(z) S_{\text {par }}^{-1}(z) \quad \text { for } \quad z \in \mathbb{C} \backslash \Sigma_{R},
$$

where the contour $\Sigma_{R}$ is obtained from $\Sigma_{1}$ for $S$ by adding the boundaries $O_{a}$ and $O_{b}$, that is, $\Sigma_{R}=\Sigma_{1} \cup \partial O_{a} \cup \partial O_{b}$. Then $S$ is the solution of the problem (33) if and only if $R$ solves the following Riemann-Hilbert problem:

$$
\begin{aligned}
& R \in H_{2 \times 2}^{2}\left(\overline{\mathbb{C}} \backslash \Sigma_{R}\right), \\
& R_{+}=R_{-} \nu_{R}=R_{-}\left\{\begin{array}{l}
N D N^{-1} \quad \text { on } \Delta^{+} \cup \Delta^{-} \backslash\left\{\Delta_{a}^{+} \cup \Delta_{b}^{+} \cup \Delta_{a}^{-} \cup \Delta_{b}^{-}\right\}, \\
U_{\alpha} N^{-1} \quad \text { on } \partial O_{\alpha}, \alpha=a, b, \\
N J N^{-1} \quad \text { on } F_{a} \cup F_{b} \backslash\left\{F_{a}^{\varepsilon} \cap F_{b}^{\varepsilon}\right\}, \\
R(z)=I+O(1 / z), \quad z \rightarrow \infty .
\end{array}\right.
\end{aligned}
$$

The jump matrices $\nu_{R}$ converge to the identity matrix. Furthermore, if we consider the matrix

$$
\Delta_{R}:=\nu_{R}-I,
$$


there are positive constants $c_{1}, C_{1}$ such that on the contour $\Sigma_{R}$, the matrices $\Delta_{R}$ satisfy the following estimates:

$$
\left\|\Delta_{R}\right\|_{L^{1}\left(\partial O_{a} \cup \partial O_{b}\right)}+\left\|\Delta_{R}\right\|_{L^{2}\left(\partial O_{a} \cup \partial O_{b}\right)}+\left\|\Delta_{R}\right\|_{L^{\infty}\left(\partial O_{a} \cup \partial O_{b}\right)} \leq \frac{c_{1}}{n},
$$

$$
\left\|\Delta_{R}\right\|_{L^{1}\left(\Sigma_{R} \backslash\left\{\partial O_{a} \cup \partial O_{b}\right\}\right.}+\left\|\Delta_{R}\right\|_{L^{2}\left(\Sigma_{R} \backslash\left\{\partial O_{a} \cup \partial O_{b}\right\}\right.}+\left\|\Delta_{R}\right\|_{L^{\infty}\left(\Sigma_{R} \backslash\left\{\partial O_{a} \cup \partial O_{b}\right\}\right.} \leq C_{1} e^{-n c_{1}} .
$$

Indeed, (69) can be verified using (66) and (65). The relation (70) follows from the fact that $N$ and $N^{-1}$ are uniformly bounded outside the neighbourhoods $O_{a}$ and $O_{b}$, while the matrices $D$ and $J$ converge to the identity matrix at a geometric rate outside these neighbourhoods (see Subsection 2.5).

Remark 1. Due to the local conditions on the density of the equilibrium measure, the matrix functions $D$ and $J$ can be analytically continued from the banks of $\Delta$ into the lens-shaped domains $d^{+}$and $d^{-}$(see Subsection 2.4). Therefore the estimate (70) holds in some neighbourhood of the corresponding contours with constants that depend on the distance to the contours.

In view of the above remark, the estimates (69), (70) allow us to conclude that

$$
R(z) \rightarrow I \quad \text { as } n \rightarrow \infty \quad \text { in } \quad \overline{\mathbb{C}} .
$$

Indeed, consider the trivial factorization $\nu_{R}=\left(b_{R}\right)_{-}^{-1}\left(b_{R}\right)_{+}$, where $\left(b_{R}\right)_{-}=I$ and $\left(b_{R}\right)_{+}=\nu_{R}$. Let us use the notation $\left(w_{R}\right)_{-}:=\left(b_{R}\right)_{-}-I=0,\left(w_{R}\right)_{+}:=\left(b_{R}\right)_{+}-I=\Delta_{R}$. Let $C$ be the Cauchy operator on the contour $\Sigma$, that is:

$$
(C f)(z):=\frac{1}{2 \pi i} \int_{\Sigma} \frac{f(s)}{s-z} d s .
$$

Then for $f \in L^{2}(\Sigma)$ we have

1. $C f$ is analytic in $\mathbb{C} \backslash \Sigma$.

2. $C f(\cdot \pm i \varepsilon)$ converges in $L^{2}(\Sigma)$ to $(C f)_{ \pm}$as $\varepsilon \searrow 0$.

3. The operator $C_{ \pm} f:=(C f)_{ \pm}$is a bounded operator from $L^{2}(\Sigma)$ into $L^{2}(\Sigma)$, and furthermore $C_{+}-C_{-}=\mathbf{1}$.

The proofs of these facts follow from the standard calculus for the Cauchy transform; we note that the boundary condition in 3 is the Sokhotskii-Plemelj formula.

Let us set

$$
C_{\Delta_{R}}(f):=C_{-}\left(f \Delta_{R}\right)=C_{-}\left(f\left(w_{R}\right)_{+}\right)+C_{+}\left(f\left(w_{R}\right)_{-}\right) .
$$

It is known (see [22]) that in view of (69), (70), for $w_{ \pm} \in L^{\infty}(\Sigma,|d z|)$, the operator $C_{\Delta_{R}}(f)$ is a bounded operator from $L^{2}\left(\Sigma_{R}, \mathbb{C}^{2 \times 2}\right)$ into itself, with norm equal to $\left\|C_{\Delta_{R}}\right\|=O(1 / n)$. Therefore the operator $I-C_{\Delta_{R}}$ is invertible and the following expression makes sense:

$$
\mu_{R}:=\left(I-C_{\Delta_{R}}\right)^{-1}\left(C_{-} \Delta_{R}\right) \in L^{2}\left(\Sigma_{R}\right) .
$$

Thus the solution $R$ of problem (68) has the integral representation

$$
R(z)=I+\int_{\Sigma_{R}} \frac{\Delta_{R}(s)+\mu_{R}(s) \Delta_{R}(s)}{s-z} \frac{d s}{2 \pi i} .
$$

From this follows (71), which as a result allows us, by inverting the chain of transformations $R \rightarrow S \rightarrow T \rightarrow Y$, to obtain the leading term of the asymptotics. 
2.9. An asymptotic expansion for $R$. We now move on to obtaining the main result of this section. Namely, we show how, using (174), as well as the asymptotic representation in powers of $1 / n$ of the matrix $\Delta_{R}$,

$$
\Delta_{R}(z) \sim \sum_{k=1}^{\infty} \frac{\Delta_{R}^{k}(z)}{n^{k}} \quad \text { as } \quad n \rightarrow 0, \quad z \in \Sigma_{R}
$$

we can explicitly compute an asymptotic expansion for $R$.

First we give an exact expression for $\Delta_{R}^{k}(z)$. On the contours $\Sigma_{R} \backslash\left\{\partial O_{a} \cup \partial O_{b}\right\}$, in view of the geometric rate of decay, $\Delta_{R}^{k}(z)=0$, where $k=1,2, \ldots$. From the definition,

$$
\Delta_{R}=\left\{\begin{array}{lll}
U_{a} N^{-1}-I & \text { on } \partial O_{a} \\
U_{b} N^{-1}-I & \text { on } \partial O_{b} .
\end{array}\right.
$$

Substituting in the expressions for $U_{a}$ and $U_{b}$, we obtain for each $k=1,2, \ldots$

$$
\begin{aligned}
\Delta_{R}^{k}= & U_{b} N^{-1}-I:=V_{b} A^{-1} N^{-1}-I=E(z) \Psi\left(\tilde{c}_{n} \zeta\right) A^{-1} N^{-1}-I \\
= & e^{\pi i / 12} N S_{0}\left(\begin{array}{cc}
e^{-i \pi / 6} & e^{i \pi / 3} \\
-e^{-i \pi / 6} & -e^{4 i \pi / 3}
\end{array}\right)^{-1}\left(\begin{array}{cc}
(-1)^{k} s_{k} & s_{k} \\
-(-1)^{k} t_{k} & t_{k}
\end{array}\right) \\
& \times\left(\begin{array}{cc}
e^{-i \pi / 4} & 0 \\
0 & e^{i \pi / 4}
\end{array}\right) S_{0}^{-1} N^{-1}\left(\frac{1}{\left(\tilde{\ell}^{b}\right)^{k}}\right), \quad z \in \partial O_{b},
\end{aligned}
$$

while for the boundary of the other domain we have

$$
\begin{aligned}
\Delta_{R}^{k}= & U_{a} N^{-1}-I:=V_{a} A^{-1} N^{-1}-I=\bar{E}(z) \bar{\Psi}\left(\tilde{c}_{n} \tilde{\zeta}\right) A^{-1} N^{-1}-I \\
= & e^{\pi i / 12} N S_{0} \sigma_{3}\left(\begin{array}{cc}
e^{-i \pi / 6} & e^{i \pi / 3} \\
-e^{-i \pi / 6} & -e^{4 i \pi / 3}
\end{array}\right)^{-1}\left(\begin{array}{rr}
(-1)^{k} s_{k} & s_{k} \\
-(-1)^{k} t_{k} & t_{k}
\end{array}\right) \sigma_{3} \\
& \times\left(\begin{array}{cc}
e^{-i \pi / 4} & 0 \\
0 & e^{i \pi / 4}
\end{array}\right) S_{0}^{-1} N^{-1}\left(\frac{1}{\left(\tilde{\ell^{a}}\right)^{k}}\right), \quad z \in \partial O_{a} .
\end{aligned}
$$

Below we will need the following result:

Lemma 1. (a) For any $k, \Delta_{R}^{k}(z)$ admits a meromorphic continuation from the boundary $\partial O_{b}$ into $O_{b}$. This continuation is analytic apart from the point $b$, where the function $\Delta_{R}^{k}(z)$ has a singularity of order at most $[(3 k+1) / 2]$.

(b) A similar statement holds for $\Delta_{R}^{k}(z)$ in $O_{a}$.

Proof. (a) As in the proof of Proposition 1, we show that $\Delta_{R}^{k}$ does not make a jump on $\Delta$ for any $k$, and then compute the order of the singularity at the point $b$.

Thus, for $k=2 j, j=1,2, \ldots$, multiplying all the matrices in (76), we have

$$
\Delta_{R}^{2 j}=\frac{1}{2}\left(\begin{array}{cc}
s_{2 j} a_{11}+t_{2 j} b_{11} & i s_{2 j} a_{12}-i t_{2 j} b_{12} \\
-i s_{2 j} a_{21}+i t_{2 j} b_{21} & s_{2 j} a_{22}+t_{2 j} b_{22}
\end{array}\right)\left(\frac{1}{\left(\tilde{\ell^{b}}\right)^{2 j}}\right),
$$

where

$$
\begin{aligned}
& a_{11}(s, \beta)=1+\frac{1}{4 \beta^{2} s^{2}}-\frac{\beta^{2}}{4 s^{2}}-\frac{s^{2}}{4 \beta^{2}}+\frac{1}{4} \beta^{2} s^{2}, \\
& b_{11}(s, \beta)=1-\frac{1}{4 \beta^{2} s^{2}}+\frac{\beta^{2}}{4 s^{2}}+\frac{s^{2}}{4 \beta^{2}}-\frac{1}{4} \beta^{2} s^{2}, \\
& a_{22}(s, \beta)=1-\frac{1}{4 \beta^{2} s^{2}}+\frac{\beta^{2}}{4 s^{2}}+\frac{s^{2}}{4 \beta^{2}}-\frac{1}{4} \beta^{2} s^{2}, \\
& b_{22}(s, \beta)=1+\frac{1}{4 \beta^{2} s^{2}}-\frac{\beta^{2}}{4 s^{2}}-\frac{s^{2}}{4 \beta^{2}}+\frac{1}{4} \beta^{2} s^{2},
\end{aligned}
$$




$$
\begin{aligned}
& a_{21}(s, \beta)=\frac{1}{2 s^{2}}+\frac{1}{4 \beta^{2} s^{2}}+\frac{\beta^{2}}{4 s^{2}}+\frac{1}{2} s^{2}-\frac{s^{2}}{4 \beta^{2}}-\frac{1}{4} \beta^{2} s^{2} \\
& b_{21}(s, \beta)=\frac{1}{2 s^{2}}+\frac{1}{4 \beta^{2} s^{2}}+\frac{\beta^{2}}{4 s^{2}}+\frac{1}{2} s^{2}-\frac{s^{2}}{4 \beta^{2}}-\frac{1}{4} \beta^{2} s^{2} \\
& a_{12}(s, \beta)=\frac{1}{2 s^{2}}-\frac{1}{4 \beta^{2} s^{2}}-\frac{\beta^{2}}{4 s^{2}}+\frac{1}{2} s^{2}+\frac{s^{2}}{4 \beta^{2}}+\frac{1}{4} \beta^{2} s^{2}, \\
& b_{12}(s, \beta)=\frac{1}{2 s^{2}}-\frac{1}{4 \beta^{2} s^{2}}-\frac{\beta^{2}}{4 s^{2}}+\frac{1}{2} s^{2}-\frac{s^{2}}{4 \beta^{2}}-\frac{1}{4} \beta^{2} s^{2},
\end{aligned}
$$

recalling that $s$ is defined in (57), (17), while $\beta$ is defined in (77).

Using the boundary conditions on $\Delta$ for $s$ and $\beta$ : $s_{+}=1 / s_{-}, \beta_{+}=i \beta_{-}$, it is easy to check that none of the elements $a_{l, m}$ or $b_{l, m}, l, m \in\{1,2\}$, changes as we pass through $\Delta$. Since $\left(\ell_{+}^{\tilde{b}}\right)^{2 j}=\left(\ell_{-}^{\tilde{b}}\right)^{2 j}$, the $\Delta_{R}^{2 j}$ do not change as we traverse $\Delta$.

We also have to check that for odd $k=2 j-1, j=1,2, \ldots, \Delta_{R}^{2 j-1}$ does not change as we go through $\Delta$ :

$$
\Delta_{R}^{2 j-1}(z)=\frac{1}{2}\left(\begin{array}{cc}
-s_{2 j-1} \tilde{a}_{11}-t_{2 j-1} \tilde{b}_{11} & i s_{2 j-1} \tilde{a}_{12}+i t_{2 j-1} \tilde{b}_{12} \\
i s_{2 j-1} \tilde{a}_{21}+i t_{2 j-1} \tilde{b}_{21} & s_{2 j-1} \tilde{a}_{22}+t_{2 j-1} \tilde{b}_{22}
\end{array}\right)\left(\frac{1}{\left(\tilde{\ell^{b}}\right)^{2 j-1}}\right)
$$

where

$$
\begin{aligned}
& \tilde{a}_{11}=\frac{1}{2 \beta^{2}}+\frac{\beta^{2}}{2}+\frac{1}{4 \beta^{2} s^{2}}-\frac{\beta^{2}}{4 s^{2}}+\frac{s^{2}}{4 \beta^{2}}-\frac{1}{4} \beta^{2} s^{2} \\
& \tilde{b}_{11}=\frac{1}{2 \beta^{2}}+\frac{\beta^{2}}{2}-\frac{1}{4 \beta^{2} s^{2}}+\frac{\beta^{2}}{4 s^{2}}-\frac{s^{2}}{4 \beta^{2}}+\frac{1}{4} \beta^{2} s^{2} \\
& \tilde{a}_{22}=\frac{1}{2 \beta^{2}}+\frac{\beta^{2}}{2}+\frac{1}{4 \beta^{2} s^{2}}-\frac{\beta^{2}}{4 s^{2}}+\frac{s^{2}}{4 \beta^{2}}-\frac{1}{4} \beta^{2} s^{2} \\
& \tilde{b}_{22}=\frac{1}{2 \beta^{2}}+\frac{\beta^{2}}{2}-\frac{1}{4 \beta^{2} s^{2}}+\frac{\beta^{2}}{4 s^{2}}-\frac{s^{2}}{4 \beta^{2}}+\frac{1}{4} \beta^{2} s^{2} \\
& \tilde{a}_{21}=\frac{1}{2 \beta^{2}}-\frac{\beta^{2}}{2}+\frac{1}{2 s^{2}}+\frac{1}{4 \beta^{2} s^{2}}+\frac{\beta^{2}}{4 s^{2}}-\frac{s^{2}}{2}+\frac{s^{2}}{4 \beta^{2}}+\frac{\beta^{2} s^{2}}{4} \\
& \tilde{b}_{21}=\frac{1}{2 \beta^{2}}-\frac{\beta^{2}}{2}-\frac{1}{2 s^{2}}-\frac{1}{4 \beta^{2} s^{2}}-\frac{\beta^{2}}{4 s^{2}}+\frac{s^{2}}{2}-\frac{s^{2}}{4 \beta^{2}}-\frac{\beta^{2} s^{2}}{4} \\
& \tilde{a}_{12}=\frac{1}{2 \beta^{2}}-\frac{\beta^{2}}{2}-\frac{1}{2 s^{2}}+\frac{1}{4 \beta^{2} s^{2}}+\frac{\beta^{2}}{4 s^{2}}+\frac{s^{2}}{2}+\frac{s^{2}}{4 \beta^{2}}+\frac{\beta^{2} s^{2}}{4} \\
& \tilde{b}_{12}=\frac{1}{2 \beta^{2}}-\frac{\beta^{2}}{2}+\frac{1}{2 s^{2}}-\frac{1}{4 \beta^{2} s^{2}}-\frac{\beta^{2}}{4 s^{2}}-\frac{s^{2}}{2}-\frac{s^{2}}{4 \beta^{2}}-\frac{\beta^{2} s^{2}}{4}
\end{aligned}
$$

Using the boundary conditions on $\Delta$ for $s$ and $\beta$ : $s_{+}=1 / s_{-}, \beta_{+}=i \beta_{-}$, it is easily checked that as we cross $\Delta,\left(\tilde{a}_{l, m}\right)_{+}=-\left(\tilde{a}_{l, m}\right)_{-}$and $\left(\tilde{b}_{l, m}\right)_{+}=\left(\tilde{b}_{l, m}\right)_{-}, l, m \in\{1,2\}$. Since $\left(\tilde{\ell}_{+}^{\tilde{b}}\right)^{2 j-1}=-\left(\tilde{\ell}_{-}^{\tilde{b}}\right)^{2 j-1}$, the $\Delta_{R}^{2 j-1}$ do not change as we pass through $\Delta$.

It remains to determine the nature of singularity at the point $b$. Each of the functions $a_{l, m}, b_{l, m}, \tilde{a}_{l, m}, \tilde{a}_{l, m}, l, m \in\{1,2\}$, has a pole of order $3 / 2$ at $b$. Indeed, in a neighbourhood of $b$ we have: $s^{2} \simeq 1 / s^{2} \simeq 1+O\left((z-b)^{1 / 2}\right)$ and $\beta^{2} \simeq(z-b)^{1 / 2}$, so that the maximal order is achieved with a term of the form $s^{2} / \beta^{2} \simeq z^{-1 / 2}$. We know that $\tilde{\ell}^{b} \simeq(z-b)^{3 / 2}$ in a neighbourhood of the point $b$. Multiplying out, we see that $\Delta_{R}^{k}$ has a pole of order $(3 k+1) / 2$.

(b) is proved similarly to (a). We will only write out the expressions for $\Delta_{R}^{2 j}$ and $\Delta_{R}^{2 j-1}$ in a neighbourhood of the point $a$. 
Thus, for $k=2 j, j=1,2, \ldots$, multiplying out all the matrices in (77), we have

$$
\Delta_{R}^{2 j}(z)=\frac{1}{2}\left(\begin{array}{cc}
s_{2 j} b_{11}+t_{2 j} a_{11} & -i s_{2 j} a_{12}+i t_{2 j} b_{12} \\
i s_{2 j} a_{21}-i t_{2 j} b_{21} & s_{2 j} b_{22}+t_{2 j} a_{22}
\end{array}\right)\left(\frac{1}{\left(\tilde{\ell^{a}}\right)^{2 j}}\right) .
$$

For odd $k=2 j-1, j=1,2, \ldots$, we have

$$
\Delta_{R}^{2 j-1}(z)=\frac{1}{2}\left(\begin{array}{cc}
-s_{2 j-1} \tilde{b}_{11}-t_{2 j-1} \tilde{a}_{11} & i s_{2 j-1} \tilde{b}_{12}+i t_{2 j-1} \tilde{a}_{12} \\
i s_{2 j-1} \tilde{b}_{21}+i t_{2 j-1} \tilde{a}_{21} & s_{2 j-1} \tilde{b}_{22}+t_{2 j-1} \tilde{a}_{22}
\end{array}\right)\left(\frac{1}{\left(\tilde{\ell^{a}}\right)^{2 j-1}}\right) .
$$

Remark 2. Observe that for $s=1$ we have

$$
\begin{aligned}
& a_{11}=1, \quad b_{11}=1, \quad a_{22}=1, \quad b_{22}=1, \\
& a_{21}=1, \quad b_{21}=1, \quad a_{12}=1, \quad b_{12}=1
\end{aligned}
$$

and

$$
\begin{aligned}
& \tilde{a}_{11}=\frac{1}{\beta^{2}}, \quad \tilde{b}_{11}=\beta^{2}, \quad \tilde{a}_{22}=\frac{1}{\beta^{2}}, \quad \tilde{b}_{22}=\beta^{2}, \\
& \tilde{a}_{21}=\frac{1}{\beta^{2}}, \quad \tilde{b}_{21}=-\beta^{2}, \quad \tilde{a}_{12}=\frac{1}{\beta^{2}}, \quad \tilde{b}_{12}=-\beta^{2} .
\end{aligned}
$$

Now (78) - (81) become the formulae given in [20] if we substitute $s=1$ in them.

Using the arguments of Theorem 7.10 in 20), from Lemma 1 we obtain

Proposition 3 (Compare with Theorem 7.10 in [20). The unique solution of the problem (68) has an asymptotic expansion in powers of $1 / n$ that is uniform in $z$ and is given by

$$
R(z) \sim I+\sum_{k=1}^{\infty} \frac{r_{k}(z)}{n^{k}}, \quad n \rightarrow \infty .
$$

Here the functions $r_{k}(z)$ are analytic in $\mathbb{C} \backslash\left\{\partial O_{a} \cup \partial O_{b}\right\}$ and can be computed explicitly.

2.10. Recurrence relations for $r_{k}$. To obtain explicit expressions for the coefficients $r_{k}$, we derive for them recurrent boundary value problems. Here we follow [24].

Thus, the jump condition for $R$ on the contour $\Sigma_{R}$ has the form

$$
R_{+}=R_{-}\left(I+\Delta_{R}\right) .
$$

Substituting the asymptotic expressions (75) and (82) for $\Delta_{R}$ and $R$, respectively, into this equality and collecting the like terms in powers of $1 / n$, we obtain for $k=1,2, \ldots$

$$
r_{k,+}(s)-r_{k,-}(s)=\sum_{j=1}^{k} r_{k-j,-}(s) \Delta_{R}^{j}(s) \quad \text { for } \quad s \in \partial O_{a} \cup \partial O_{b},
$$

where $r_{0,-}=I$. In particular, taking into account that $r_{k}(z)=O(1 / z)$ as $z \rightarrow \infty$, and that $\Delta_{R}^{k}(s)=0$ for $s \in \Sigma_{R}$, we can obtain from this formula, using the Sokhotskii-Plemelj formula, the integral representation

$$
r_{k}(z)=\frac{1}{2 \pi i} \int_{\Sigma_{R}} \sum_{j=1}^{k} r_{k-j,-}(s) \Delta_{R}^{j}(s) \frac{d s}{s-z} \quad \text { for } \quad z \in \mathbb{C} \backslash \Sigma_{R} .
$$

From equation (84) we can also obtain additive Riemann-Hilbert boundary value problems. For example, we write them down for $r_{1}$ and $r_{2}$ :

$$
\begin{gathered}
r_{1} \in H_{2 \times 2}^{2}\left(\mathbb{C} \backslash\left(\partial O_{a} \cup \partial O_{b}\right)\right), \\
r_{1,+}(s)-r_{1,-}(s)=\Delta_{R}^{1}(s) \quad \text { on } \quad s \in \partial O_{a} \cup \partial O_{b}, \\
r_{1}(z)=O\left(\frac{1}{z}\right), \quad z \rightarrow \infty .
\end{gathered}
$$


Knowing $r_{k}$, we can pose a similar problem for $r_{k+1}$ :

$$
\begin{gathered}
r_{2} \in H_{2 \times 2}^{2}\left(\mathbb{C} \backslash\left(\partial O_{a} \cup \partial O_{b}\right)\right), \\
r_{2,+}(s)-r_{2,-}(s)=r_{1,-}(s) \Delta_{R}^{1}(s)+\Delta_{R}^{2}(s) \quad \text { on } \quad s \in \partial O_{a} \cup \partial O_{b}, \\
\\
r_{2}(z)=O\left(\frac{1}{z}\right), \quad z \rightarrow \infty,
\end{gathered}
$$

and so on.

2.11. The asymptotics of orthogonal polynomials with respect to a weight function $W_{n}$ (the completion of the proof of Theorem 1). In this subsection we return to the orthogonal polynomials. "Unwinding" the chain of transformations

$$
Y \rightarrow T \rightarrow S \rightarrow R
$$

in the opposite direction and using the asymptotic expansion for $R$ obtained in the previous subsection, we will obtain an asymptotic expansion for $Y$.

Let $K$ be a closed set in $\overline{\mathbb{C}} \backslash \Delta$. Then we can choose both the arcs $\Delta^{ \pm}$and the disks $\partial O_{a}$ and $\partial O_{b}$ so that they do not intersect $K$ and, in addition, $K$ lies on their +-side.

From the definition of the matrix functions $T, S, R$ in $K$ we have

$$
S(z)=R(z) N(z), \quad T(z)=S(z), \quad Y(z)=C^{-1}(z) T(z) \Phi^{-1}(z) .
$$

Therefore

$$
Y(z)=C^{-1} R(z) N(z) \Phi^{-1}(z), \quad z \in K .
$$

Substituting the expressions for the matrices $C$ and $\Phi$ found in Subsection 2.3, we obtain that for $z \in K$

$$
\begin{aligned}
Y(z)=\operatorname{diag}\left\{C_{n}, C_{n}^{-1}\right\}\left(\begin{array}{cc}
R_{11}(z) & R_{12}(z) \\
R_{21}(z) & R_{22}(z)
\end{array}\right) \\
\times\left(\begin{array}{cc}
\frac{\beta+\beta^{-1}}{2} & \frac{\beta-\beta^{-1}}{2 i} \\
-\frac{\beta-\beta^{-1}}{2 i} & \frac{\beta+\beta^{-1}}{2}
\end{array}\right) \operatorname{diag}\left\{\varphi(z), \varphi^{-1}(z)\right\},
\end{aligned}
$$

where the function $\varphi$ and the constant $C_{n}$ were defined in (18), while $\beta$ is given by (7). Therefore

$$
Y_{11}(z):=P_{n}(z)=\frac{c_{n} \varphi(z)}{2}\left[R_{11}(z)\left(\beta(z)+\beta^{-1}(z)\right)+i R_{12}(z)\left(\beta(z)-\beta^{-1}(z)\right)\right],
$$

while for functions of the second kind we have

$$
\begin{aligned}
Y_{12}(z): & =R_{n}(z) \\
& =\frac{c_{n}}{2 \varphi(z)}\left[i R_{11}(z)\left(-\beta(z)+\beta^{-1}(z)\right)+R_{12}(z)\left(\beta(z)+\beta^{-1}(z)\right)\right] .
\end{aligned}
$$

Substituting the asymptotic expansions (82) for $R_{11}$ and $R_{12}$, we have:

$$
\begin{aligned}
\frac{2 P_{n}(z)}{c_{n} \varphi(z)} & \sim\left(\beta(z)+\beta^{-1}(z)\right)\left[1+\sum_{k=1}^{\infty} \frac{r_{k}^{11}(z)}{n^{k}}+i \frac{\beta(z)-\beta^{-1}(z)}{\beta(z)+\beta^{-1}(z)} \sum_{k=1}^{\infty} \frac{r_{k}^{12}(z)}{n^{k}}\right] \\
& =\left(\beta(z)+\beta^{-1}(z)\right)\left[1+\sum_{k=1}^{\infty}\left(r_{k}^{11}(z)+i \frac{\beta(z)-\beta^{-1}(z)}{\beta(z)+\beta^{-1}(z)} r_{k}^{12}(z)\right) \frac{1}{n^{k}}\right],
\end{aligned}
$$

where

$$
r_{k}(z)=\left(\begin{array}{cc}
r_{k}^{11}(z) & r_{k}^{12}(z) \\
r_{k}^{21}(z) & r_{k}^{22}(z)
\end{array}\right)
$$


Thus we have proved Theorem 1 with

$$
\Pi_{k}(z)=r_{k}^{11}(z)+i \frac{\beta(z)-\beta^{-1}(z)}{\beta(z)+\beta^{-1}(z)} r_{k}^{12}(z), \quad k=1,2, \ldots,
$$

where the $r_{k}$ are defined by (84)-87).

\section{The Computation of $\Pi_{1}$}

From Lemma 1 we know that $\Delta_{R}^{1}$ is analytic in a neighbourhood of the points $a$ and $b$, apart from having poles of order 2 at these points. Therefore

$$
\begin{aligned}
& \Delta_{R}^{1}(z)=\frac{\tilde{A}^{(2)}}{(z-b)^{2}}+\frac{\tilde{A}^{(1)}}{(z-b)}+O(1) \quad \text { as } \quad z \rightarrow b, \\
& \Delta_{R}^{1}(z)=\frac{\tilde{B}^{(2)}}{(z-a)^{2}}+\frac{\tilde{B}^{(1)}}{(z-a)}+O(1) \quad \text { as } \quad z \rightarrow a
\end{aligned}
$$

for some matrices $\tilde{A}^{(1)}, \tilde{A}^{(2)}, \tilde{B}^{(1)}$ and $\tilde{B}^{(2)}$. Therefore it is obvious that the function

$$
r_{1}(z)= \begin{cases}\frac{\tilde{A}^{(2)}}{(z-b)^{2}}+\frac{\tilde{A}^{(1)}}{(z-b)} \frac{\tilde{B}^{(2)}}{(z-a)^{2}}+\frac{\tilde{B}^{(1)}}{(z-b)} & \text { for } \quad z \in \mathbb{C} \backslash\left(\overline{O_{b}} \cup \overline{O_{a}}\right), \\ \frac{\tilde{A}^{(2)}}{(z-b)^{2}}+\frac{\tilde{A}^{(1)}}{(z-b)} \frac{\tilde{B}^{(2)}}{(z-a)^{2}}+\frac{\tilde{B}^{(1)}}{(z-b)}-\Delta_{R}^{1} & \text { for } \quad z \in O_{b} \cup O_{a},\end{cases}
$$

solves the problem (86).

From Lemma 1 it follows that in a neighbourhood of the point $b$

$$
\Delta_{R}^{1}(z)=\frac{1}{2}\left(\begin{array}{cc}
-s_{1} \tilde{a}_{11}(s, \beta)-t_{1} \tilde{b}_{11}(s, \beta) & i s_{1} \tilde{a}_{12}(s, \beta)+i t_{1} \tilde{b}_{12}(s, \beta) \\
i s_{1} \tilde{a}_{21}(s, \beta)+i t_{1} \tilde{b}_{21}(s, \beta) & s_{1} \tilde{a}_{22}(s, \beta)+t_{1} \tilde{b}_{22}(s, \beta)
\end{array}\right)\left(\frac{1}{\left(\tilde{\ell^{b}}\right)}\right),
$$

while in a neighbourhood of the point $a$

$$
\Delta_{R}^{1}(z)=\frac{1}{2}\left(\begin{array}{cc}
-s_{1} \tilde{b}_{11}(s, \beta)-t_{1} \tilde{a}_{11}(s, \beta) & i s_{1} \tilde{b}_{12}(s, \beta)+i t_{1} \tilde{a}_{12}(s, \beta) \\
i s_{1} \tilde{b}_{21}(s, \beta)+i t_{1} \tilde{a}_{21}(s, \beta) & s_{1} \tilde{b}_{22}(s, \beta)+t_{1} \tilde{a}_{22}(s, \beta)
\end{array}\right)\left(\frac{1}{\left(\tilde{\ell^{a}}\right)}\right) .
$$

We recall the expressions for $\beta^{2}$ and $\tilde{\ell^{b}}$ :

$$
\begin{aligned}
& \beta^{2}(z)=\frac{(z-b)^{1 / 2}}{(z-a)^{1 / 2}}, \\
& \tilde{\ell^{b}}(z)=i \pi \int_{z}^{b} d \lambda(x) .
\end{aligned}
$$

For the derivative of the equilibrium measure $\lambda$ in an external field under the hypotheses of the theorem we have assumed that

$$
\lambda^{\prime}(z)=\frac{1}{2 \pi} \sqrt{(z-a)(z-b)} h(z) .
$$

Substituting the expression for the derivative of the equilibrium measure into the integral for $\tilde{\ell^{b}}(z)$, we obtain

$$
\tilde{\ell^{b}}(z)=\frac{i}{2} \int_{z}^{b} \sqrt{(x-a)(x-b)} h(x) d x .
$$

Let us obtain an expansion in a neighbourhood of the point $b$. To that end, we write out the series in powers of $(x-b)$ in a neighbourhood of the point $b$ for the functions under 
the integral sign:

$$
\begin{gathered}
\sqrt{(x-a)}=\sqrt{(b-a)}+\frac{1}{2} \frac{1}{\sqrt{b-a}}(x-b)-\frac{1}{8} \frac{1}{(b-a)^{3 / 2}}(x-b)^{2}+\cdots, \\
h(x)=h(b)+h^{\prime}(b)(x-b)+\frac{1}{2} h^{(2)}(b)(x-b)^{2}+\cdots
\end{gathered}
$$

so that

$$
\begin{aligned}
\tilde{\ell^{b}}(z)= & \frac{i}{2} \int_{z}^{b} \sqrt{(x-b)} \sqrt{b-a} h(b) d x \\
& +\frac{i}{2} \int_{z}^{b} \sqrt{(x-b)}(x-b)\left(\frac{1}{2} \frac{1}{\sqrt{b-a}} h(b)+\sqrt{b-a} h^{\prime}(b)\right) d x+\cdots
\end{aligned}
$$

Integrating, we obtain

$$
\begin{aligned}
\tilde{\ell^{b}}(z)=\frac{i}{2}( & -\frac{2}{3} \sqrt{b-a} h(b)(z-b)^{3 / 2} \\
& \left.\quad-\frac{2}{5}\left(\frac{1}{2} \frac{1}{\sqrt{b-a}} h(b)+\sqrt{b-a} h^{\prime}(b)\right)(z-b)^{5 / 2}-\cdots\right) \\
= & -\frac{i}{2}(z-b)^{3 / 2}\left(\frac{2}{3} \sqrt{b-a} h(b)+\frac{2}{5}\left(\frac{1}{2} \frac{1}{\sqrt{b-a}} h(b)+\sqrt{b-a} h^{\prime}(b)\right)(z-b)+\cdots\right) .
\end{aligned}
$$

Therefore the expansion of $1 / \tilde{\ell^{b}}$ in powers of $(z-b)$ will have the form

$$
\begin{aligned}
\frac{1}{\tilde{\ell}^{b}(z)}= & \frac{2 i}{(z-b)^{3 / 2}} \\
& \times\left(\frac{3}{2} \frac{1}{\sqrt{b-a} h(b)}-\frac{9}{10} \frac{\frac{1}{2} \frac{1}{\sqrt{b-a}} h(b)+\sqrt{b-a} h^{\prime}(b)}{(b-a) h^{2}(b)}(z-b)+O\left((z-b)^{2}\right)\right) .
\end{aligned}
$$

Now we turn to the other endpoint of the support, the point $a$. There we have

$$
\tilde{\ell^{a}}(z)=i \pi \int_{a}^{z} d \lambda(x)=\frac{i}{2} \int_{a}^{z} \sqrt{(x-a)(x-b)} h(x) d x .
$$

We write down the power series for the functions under the integral sign:

$$
\begin{aligned}
\sqrt{(x-b)} & =\sqrt{(a-b)}+\frac{1}{2} \frac{1}{\sqrt{a-b}}(x-a)-\frac{1}{8} \frac{1}{(a-b)^{3 / 2}}(x-a)^{2}+\cdots, \\
h(x) & =h(a)+h^{\prime}(a)(x-a)+\frac{1}{2} h^{(2)}(a)(x-a)^{2}+\cdots
\end{aligned}
$$

so that

$$
\begin{aligned}
\tilde{\ell^{a}}(z)= & \frac{i}{2} \int_{a}^{z} \sqrt{(x-a)} \sqrt{(a-b)} h(a) d x \\
& +\frac{i}{2} \int_{a}^{z} \sqrt{(x-a)}(x-a)\left(\frac{1}{2} \frac{1}{\sqrt{(a-b)}} h(a)+\sqrt{(a-b)} h^{\prime}(a)\right) d x+\cdots
\end{aligned}
$$


Integrating, we obtain

$$
\begin{aligned}
\tilde{\ell^{a}}(z)= & \frac{i}{2}\left(\frac{2}{3} \sqrt{a-b} h(a)(z-a)^{3 / 2}\right. \\
& \left.+\frac{2}{5}\left(\frac{1}{2} \frac{1}{\sqrt{a-b}} h(a)+\sqrt{a-b} h^{\prime}(a)\right)(z-a)^{5 / 2}-\cdots\right) \\
= & \frac{i}{2}(z-a)^{3 / 2}\left(\frac{2}{3} \sqrt{a-b} h(a)+\frac{2}{5}\left(\frac{1}{2} \frac{1}{\sqrt{a-b}} h(a)+\sqrt{a-b} h^{\prime}(a)\right)(z-a)+\cdots\right) .
\end{aligned}
$$

Therefore the expansion of $1 / \tilde{\ell^{a}}$ in powers of $(z-a)$ will have the form

$$
\begin{aligned}
\frac{1}{\tilde{\ell^{a}}(z)}= & -\frac{2 i}{(z-a)^{3 / 2}} \\
& \times\left(\frac{3}{2} \frac{1}{\sqrt{(a-b)} h(a)}-\frac{9}{10} \frac{\frac{1}{2} \frac{1}{\sqrt{(a-b)}} h(a)+\sqrt{(a-b)} h^{\prime}(a)}{(a-b) h^{2}(a)}(z-a)+\cdots\right) .
\end{aligned}
$$

It remains to obtain the series for $\tilde{a}_{11}, \tilde{b}_{11}, \tilde{a}_{12}, \tilde{b}_{12}, \tilde{a}_{21}, \tilde{b}_{21}, \tilde{a}_{22}$ and $\tilde{b}_{22}$.

First we will show how to obtain a power series for the Szegö function $\tilde{s}(\tilde{W} ; z)$, where $\tilde{W}=\exp \left\{-2 Q_{1}\right\}$. We know that in the class of bounded functions, $\tilde{s}(\tilde{W} ; z)$ is given by (17). We will analyse the behaviour of this function in a neighbourhood of the point $b$. We introduce a closed contour $\gamma$ which encircles the support of the equilibrium measure $\lambda$, contains a point $z \in O_{b}$ and has a counterclockwise orientation. Then we have

Lemma 2. In a neighbourhood of $b$,

$$
\tilde{s}(\tilde{W} ; z)=\frac{1}{\tilde{W}^{1 / 2}(z)} \exp \left\{-\frac{1}{2}((z-a)(z-b))^{1 / 2} \sum_{k=0}^{\infty} c_{k}(z-b)^{k}\right\},
$$

where the coefficients $c_{k}$ are given by the formula

$$
c_{k}:=\frac{1}{2 \pi i} \int_{\gamma} \frac{\log \tilde{W}(\zeta)}{((\zeta-a)(\zeta-b))^{1 / 2}} \frac{d \zeta}{(\zeta-b)^{k+1}} .
$$

Proof. Consider the integral

$$
\int_{\gamma} \frac{\log \tilde{W}(t)}{z-t} \frac{d t}{2 \pi i w_{\Delta}}
$$

The integrand has three singularities inside the contour $\gamma$, the points $a, b$ and the point $z$. We encircle these singularities by contours $\gamma_{0}$ and $\gamma_{1}$. Then by Cauchy's theorem we obtain

$$
\int_{\gamma} \frac{\log \tilde{W}(t)}{z-t} \frac{d t}{2 \pi i w_{\Delta}}+\int_{\gamma_{0}} \frac{\log \tilde{W}(t)}{z-t} \frac{d t}{2 \pi i w_{\Delta}}+\int_{\gamma_{1}} \frac{\log \tilde{W}(t)}{z-t} \frac{d t}{2 \pi i w_{\Delta}}=0 .
$$

The last integral is nothing but the residue at the point $z$. As the integrand has a simple pole at $z$, the residue is

$$
\int_{\gamma_{1}} \frac{\log \tilde{W}(t)}{z-t} \frac{d t}{2 \pi i w_{\Delta}}=\frac{1}{2 \pi i} \int_{\overline{\gamma_{1}}} \frac{\log \tilde{W}(t)}{w_{\Delta}} \frac{d t}{t-z}=\frac{\log \tilde{W}(z)}{((z-a)(z-b))^{1 / 2}},
$$

where the contour $\overline{\gamma_{1}}$ is the same as $\gamma_{1}$ but with the opposite orientation.

It is not hard to verify that

$$
\int_{\gamma_{0}} \frac{\log \tilde{W}(t)}{z-t} \frac{d t}{2 \pi i w_{\Delta}}=2 \int_{\Delta} \frac{\log \tilde{W}(t)}{z-t} \frac{d t}{2 \pi i w_{\Delta}}
$$


so that

$$
\int_{\Delta} \frac{\log \tilde{W}(t)}{z-t} \frac{d t}{2 \pi i w_{\Delta+}}=-\frac{1}{2}\left(\frac{\log \tilde{W}(z)}{((z-a)(z-b))^{1 / 2}}+\int_{\gamma} \frac{\log \tilde{W}(t)}{z-t} \frac{d t}{2 \pi i w_{\Delta}}\right) .
$$

Thus, substituting the identity (97) into the expression for the Szegö function, we obtain

$$
\tilde{s}(\tilde{W} ; z)=\frac{1}{\tilde{W}^{1 / 2}(z)} \exp \left\{-\frac{1}{2}((z-a)(z-b))^{1 / 2} \int_{\gamma} \frac{\log \tilde{W}(t)}{z-t} \frac{d t}{2 \pi i w_{\Delta}}\right\} .
$$

Next, in the formula above, the integral is a Cauchy integral, which defines an analytic function in any simply connected domain that does not contain points of the contour $\gamma$, so that at $z \in O_{b}$ it admits the expansion

$$
\int_{\gamma} \frac{\log \tilde{W}(t)}{z-t} \frac{d t}{2 \pi i w_{\Delta}}=\sum_{k=0}^{\infty} c_{k}(z-b)^{k}
$$

where the coefficients $c_{k}$ are given by (96).

Similarly we can prove the following

Lemma 3. In a neighbourhood of the point a we have

$$
\tilde{s}(\tilde{W} ; z)=\frac{1}{\tilde{W}^{1 / 2}(z)} \exp \left\{-\frac{1}{2}((z-a)(z-b))^{1 / 2} \sum_{k=0}^{\infty} d_{k}(z-a)^{k}\right\},
$$

where the coefficients $d_{k}$ are given by

$$
d_{k}:=\frac{1}{2 \pi i} \int_{\gamma} \frac{\log \tilde{W}(\zeta)}{((\zeta-a)(\zeta-b))^{1 / 2}} \frac{d \zeta}{(\zeta-a)^{k+1}} .
$$

Using these lemmas, it is easy to obtain an asymptotic expansion for $s^{2}(z)$, where $s(z):=\tilde{s}(\tilde{W} ; z) \tilde{W}^{1 / 2}(z)$. As $z \rightarrow b$, we have

$$
\begin{aligned}
s^{2}(z)= & \exp \left\{-((z-a)(z-b))^{1 / 2} \sum_{k=0}^{\infty} c_{k}(z-b)^{k}\right\} \\
= & 1-c_{0} \sqrt{(b-a)}(z-b)^{1 / 2}+\frac{1}{2} c_{0}^{2}(b-a)(z-b) \\
& -\frac{1}{6}\left(\frac{3\left(2 c_{1}(b-a)+c_{0}\right)+c_{0}^{2}(b-a)^{2}}{\sqrt{(b-a)}}\right)(z-b)^{3 / 2}-\cdots,
\end{aligned}
$$

while the expansion for $1 / s^{2}$ has the form

$$
\begin{aligned}
\frac{1}{s^{2}(z)}=1 & +c_{0} \sqrt{(b-a)}(z-b)^{1 / 2}+\frac{1}{2} c_{0}^{2}(b-a)(z-b) \\
& +\frac{1}{6}\left(\frac{3\left(2 c_{1}(b-a)+c_{0}\right)+c_{0}^{2}(b-a)^{2}}{\sqrt{(b-a)}}\right)(z-b)^{3 / 2}+\cdots
\end{aligned}
$$

In what follows it will be convenient to consider the combined series for $s^{2}+\frac{1}{s^{2}}$ and $s^{2}-\frac{1}{s^{2}}$ :

$$
\begin{aligned}
& s^{2}+\frac{1}{s^{2}}=2+c_{0}^{2}(b-a)(z-b)+\cdots, \\
& s^{2}-\frac{1}{s^{2}}=-2 c_{0} \sqrt{(b-a)}(z-b)^{1 / 2}-\frac{1}{3}\left(\frac{3\left(2 c_{1}(b-a)+c_{0}\right)+c_{0}^{2}(b-a)^{2}}{\sqrt{(b-a)}}\right)(z-b)^{3 / 2} .
\end{aligned}
$$


Now we can write down the series for $\tilde{a}_{11}, \tilde{b}_{11}, \tilde{a}_{12}, \tilde{b}_{12}, \tilde{a}_{21}, \tilde{b}_{21}, \tilde{a}_{22}$ and $\tilde{b}_{22}$ :

$$
\begin{aligned}
\tilde{a}_{11} & =\frac{1}{2 \beta^{2}}+\frac{\beta^{2}}{2}+\frac{1}{4 \beta^{2} s^{2}}-\frac{\beta^{2}}{4 s^{2}}+\frac{s^{2}}{4 \beta^{2}}-\frac{1}{4} \beta^{2} s^{2} \\
& =\frac{1}{2}\left(\frac{1}{\beta^{2}}+\beta^{2}\right)+\frac{1}{4}\left(\frac{1}{\beta^{2}}-\beta^{2}\right)\left(\frac{1}{s^{2}}+s^{2}\right) \\
\tilde{b}_{11} & =\frac{1}{2 \beta^{2}}+\frac{\beta^{2}}{2}-\frac{1}{4 \beta^{2} s^{2}}+\frac{\beta^{2}}{4 s^{2}}-\frac{s^{2}}{4 \beta^{2}}+\frac{1}{4} \beta^{2} s^{2} \\
& =\frac{1}{2}\left(\frac{1}{\beta^{2}}+\beta^{2}\right)+\frac{1}{4}\left(\beta^{2}-\frac{1}{\beta^{2}}\right)\left(\frac{1}{s^{2}}+s^{2}\right), \\
\tilde{a}_{21} & =\frac{1}{2 \beta^{2}}-\frac{\beta^{2}}{2}+\frac{1}{2 s^{2}}+\frac{1}{4 \beta^{2} s^{2}}+\frac{\beta^{2}}{4 s^{2}}-\frac{s^{2}}{2}+\frac{s^{2}}{4 \beta^{2}}+\frac{\beta^{2} s^{2}}{4} \\
& =\frac{1}{2}\left(\frac{1}{\beta^{2}}-\beta^{2}\right)-\frac{1}{2}\left(s^{2}-\frac{1}{s^{2}}\right)+\frac{1}{4}\left(\beta^{2}+\frac{1}{\beta^{2}}\right)\left(\frac{1}{s^{2}}+s^{2}\right), \\
\tilde{b}_{21} & =\frac{1}{2 \beta^{2}}-\frac{\beta^{2}}{2}-\frac{1}{2 s^{2}}-\frac{1}{4 \beta^{2} s^{2}}-\frac{\beta^{2}}{4 s^{2}}+\frac{s^{2}}{2}-\frac{s^{2}}{4 \beta^{2}}-\frac{\beta^{2} s^{2}}{4} \\
& =\frac{1}{2}\left(\frac{1}{\beta^{2}}-\beta^{2}\right)+\frac{1}{2}\left(s^{2}-\frac{1}{s^{2}}\right)-\frac{1}{4}\left(\beta^{2}+\frac{1}{\beta^{2}}\right)\left(\frac{1}{s^{2}}+s^{2}\right), \\
\tilde{a}_{12} & =\frac{1}{2 \beta^{2}}-\frac{\beta^{2}}{2}-\frac{1}{2 s^{2}}+\frac{1}{4 \beta^{2} s^{2}}+\frac{\beta^{2}}{4 s^{2}}+\frac{s^{2}}{2}+\frac{s^{2}}{4 \beta^{2}}+\frac{\beta^{2} s^{2}}{4} \\
& =\frac{1}{2}\left(\frac{1}{\beta^{2}}-\beta^{2}\right)+\frac{1}{2}\left(s^{2}-\frac{1}{s^{2}}\right)+\frac{1}{4}\left(\beta^{2}+\frac{1}{\beta^{2}}\right)\left(\frac{1}{s^{2}}+s^{2}\right), \\
\tilde{b}_{12} & =\frac{1}{2 \beta^{2}}-\frac{\beta^{2}}{2}+\frac{1}{2 s^{2}}-\frac{1}{4 \beta^{2} s^{2}}-\frac{\beta^{2}}{4 s^{2}}-\frac{s^{2}}{2}-\frac{s^{2}}{4 \beta^{2}}-\frac{\beta^{2} s^{2}}{4} \\
& =\frac{1}{2}\left(\frac{1}{\beta^{2}}-\beta^{2}\right)-\frac{1}{2}\left(s^{2}-\frac{1}{s^{2}}\right)-\frac{1}{4}\left(\beta^{2}+\frac{1}{\beta^{2}}\right)\left(\frac{1}{s^{2}}+s^{2}\right) .
\end{aligned}
$$

We also have $\tilde{a}_{22}=\tilde{a}_{11}$ and $\tilde{b}_{22}=\tilde{b}_{11}$.

Furthermore, we will be using the expressions

$$
\begin{aligned}
\frac{1}{\beta^{2}}+\beta^{2}=\frac{1}{(z-b)^{1 / 2}} & \left(\sqrt{(b-a)}+\frac{3}{2} \frac{1}{\sqrt{(b-a)}}(z-b)\right. \\
& \left.-\frac{5}{8} \frac{1}{(b-a)^{3 / 2}}(z-b)^{2}+O\left((z-b)^{3}\right)\right), \\
\frac{1}{\beta^{2}}-\beta^{2}=\frac{1}{(z-b)^{1 / 2}}( & \sqrt{(b-a)}-\frac{1}{2} \frac{1}{\sqrt{(b-a)}}(z-b) \\
+ & \left.\frac{3}{8} \frac{1}{(b-a)^{3 / 2}}(z-b)^{2}+O\left((z-b)^{3}\right)\right) .
\end{aligned}
$$

Then

$$
\begin{aligned}
& \left(s_{1} \tilde{a}_{11}+t_{1} \tilde{b}_{11}\right)=\left(s_{1} \tilde{a}_{22}+t_{1} \tilde{b}_{22}\right) \\
& \quad=\frac{1}{2}\left(\frac{1}{\beta^{2}}+\beta^{2}\right)\left(s_{1}+t_{1}\right)+\frac{1}{4}\left(\frac{1}{\beta^{2}}-\beta^{2}\right)\left(\frac{1}{s^{2}}+s^{2}\right)\left(s_{1}-t_{1}\right) \\
& \quad=\frac{1}{(z-b)^{1 / 2}}\left(\frac{5 \sqrt{(b-a)}}{72}+\frac{1}{24}\left(c_{0}^{2}(b-a)^{3 / 2}-\frac{3}{2 \sqrt{(b-a)}}\right)(z-b)+O\left((z-b)^{2}\right)\right) .
\end{aligned}
$$


Similarly,

$$
\begin{aligned}
&\left(s_{1} \tilde{a}_{12}+t_{1} \tilde{b}_{12}\right) \\
&=\frac{1}{2}\left(\frac{1}{\beta^{2}}-\beta^{2}\right)\left(s_{1}+t_{1}\right)+\left(\frac{1}{2}\left(\frac{1}{s^{2}}-s^{2}\right)+\frac{1}{4}\left(\beta^{2}+\frac{1}{\beta^{2}}\right)\left(\frac{1}{s^{2}}+s^{2}\right)\right)\left(s_{1}-t_{1}\right) \\
&=\frac{1}{(z-b)^{1 / 2}}\left(\frac{5}{72} \sqrt{(b-a)}\right. \\
&\left.\quad+\left[\frac{19}{144} \frac{1}{\sqrt{(b-a)}}+\frac{c_{0}^{2}}{24}(b-a)^{3 / 2}-\frac{1}{6} c_{0} \sqrt{(b-a)}\right](z-b)\right),
\end{aligned}
$$

and finally the last term:

$$
\begin{aligned}
&\left(s_{1} \tilde{a}_{21}+t_{1} \tilde{b}_{21}\right) \\
&=\frac{1}{2}\left(\frac{1}{\beta^{2}}-\beta^{2}\right)\left(s_{1}+t_{1}\right)+\left(-\frac{1}{2}\left(\frac{1}{s^{2}}-s^{2}\right)+\frac{1}{4}\left(\beta^{2}+\frac{1}{\beta^{2}}\right)\left(\frac{1}{s^{2}}+s^{2}\right)\right)\left(s_{1}-t_{1}\right) \\
&=\frac{1}{(z-b)^{1 / 2}}\left(\frac{5}{72} \sqrt{(b-a)}\right. \\
&\left.\quad+\left[\frac{19}{144} \frac{1}{\sqrt{(b-a)}}+\frac{c_{0}^{2}}{24}(b-a)^{3 / 2}+\frac{1}{6} c_{0} \sqrt{(b-a)}\right](z-b)\right) .
\end{aligned}
$$

Thus in a neighbourhood of the point $b$ we have

$$
\begin{aligned}
& \Delta_{R}^{1}(z)=\frac{i}{(z-b)^{2}} \\
& \times\left(\begin{array}{c}
-\left(\frac{5 \sqrt{(b-a)}}{72}+\frac{1}{24}\left(c_{0}^{2}(b-a)^{3 / 2}-\frac{3}{2 \sqrt{(b-a)}}\right)(z-b)\right) \\
i\left(\frac{5}{72} \sqrt{(b-a)}+\left\{\frac{19}{144} \frac{1}{\sqrt{(b-a)}}+\frac{c_{0}^{2}}{24}(b-a)^{3 / 2}+\frac{1}{6} c_{0} \sqrt{(b-a)}\right\}(z-b)\right)
\end{array}\right. \\
& i\left(\frac{5}{72} \sqrt{(b-a)}+\left\{\frac{19}{144} \frac{1}{\sqrt{(b-a)}}+\frac{c_{0}^{2}}{24}(b-a)^{3 / 2}-\frac{1}{6} c_{0} \sqrt{(b-a)}\right\}(z-b)\right) \\
& \left(\frac{5 \sqrt{(b-a)}}{72}+\frac{1}{24}\left(c_{0}^{2}(b-a)^{3 / 2}-\frac{3}{2 \sqrt{(b-a)}}\right)(z-b)\right) \\
& \times\left(\frac{3}{2} \frac{1}{\sqrt{b-a} h(b)}-\frac{9}{10} \frac{\frac{1}{2} \frac{1}{\sqrt{b-a}} h(b)+\sqrt{b-a} h^{\prime}(b)}{(b-a) h^{2}(b)}(z-b)+\cdots\right) \\
& =\frac{i}{(z-b)^{2}}\left(\begin{array}{cc}
-\frac{5}{48} \frac{1}{h(b)} & i \frac{5}{48 h(b)} \\
i \frac{5}{48 h(b)} & \frac{5}{48} \frac{1}{h(b)}
\end{array}\right) \\
& +\frac{i}{(z-b)}\left(\begin{array}{c}
\frac{1}{16} \frac{\frac{1}{2} h(b)+(b-a) h^{\prime}(b)}{(b-a) h^{2}(b)}-\frac{1}{16} \frac{1}{\sqrt{b-a} h(b)}\left(c_{0}^{2}(b-a)^{3 / 2}-\frac{3}{2 \sqrt{(b-a)}}\right) \\
-\frac{i}{16} \frac{\frac{1}{2} h(b)+(b-a) h^{\prime}(b)}{(b-a) h^{2}(b)}+i \frac{3}{2 h(b)}\left\{\frac{19}{144} \frac{1}{(b-a)}+\frac{c_{0}^{2}}{24}(b-a)+\frac{1}{6} c_{0}\right\}
\end{array}\right. \\
& -\frac{i}{16} \frac{\frac{1}{2} h(b)+(b-a) h^{\prime}(b)}{(b-a) h^{2}(b)}+i \frac{3}{2 h(b)}\left\{\frac{19}{144} \frac{1}{(b-a)}+\frac{c_{0}^{2}}{24}(b-a)-\frac{1}{6} c_{0}\right\} \\
& \left.-\frac{1}{16} \frac{\frac{1}{2} h(b)+(b-a) h^{\prime}(b)}{(b-a) h^{2}(b)}+\frac{1}{16} \frac{1}{\sqrt{b-a} h(b)}\left(c_{0}^{2}(b-a)^{3 / 2}-\frac{3}{2 \sqrt{(b-a)}}\right)\right) \\
& +O(1),
\end{aligned}
$$


as $z \rightarrow b$. After slight simplifications, we obtain an explicit form for the matrices $\tilde{A}^{(2)}$ and $\tilde{A}^{(1)}$ :

$$
\begin{aligned}
& \tilde{A}^{(2)}(z)=i \frac{5}{48} \frac{1}{h(b)}\left(\begin{array}{cc}
-1 & i \\
i & 1
\end{array}\right), \\
& \tilde{A}^{(1)}(z)=-\frac{i}{16} \frac{\frac{1}{2} h(b)+(b-a) h^{\prime}(b)}{(b-a) h^{2}(b)}\left(\begin{array}{cc}
-1 & i \\
i & 1
\end{array}\right) \\
& +\frac{i}{16} \frac{1}{(b-a) h(b)}\left(\begin{array}{cc}
-\left(c_{0}^{2}(b-a)^{2}-\frac{3}{2}\right) & i\left\{\frac{19}{6}+c_{0}^{2}(b-a)^{2}-4 c_{0}(b-a)\right\} \\
i\left\{\frac{19}{6}+c_{0}^{2}(b-a)^{2}+4 c_{0}(b-a)\right\} & \left(c_{0}^{2}(b-a)^{2}-\frac{3}{2}\right)
\end{array}\right) .
\end{aligned}
$$

We note that for $c_{0}=0$ the expression for $\tilde{A}^{(1)}(z)$ takes the form

$$
\tilde{A}^{(1)}(z)=-\frac{i}{16} \frac{\frac{1}{2} h(b)+(b-a) h^{\prime}(b)}{(b-a) h^{2}(b)}\left(\begin{array}{cc}
-1 & i \\
i & 1
\end{array}\right)+\frac{i}{16} \frac{1}{(b-a) h(b)}\left(\begin{array}{cc}
\frac{3}{2} & i \frac{19}{6} \\
i \frac{19}{6} & -\frac{3}{2}
\end{array}\right) .
$$

Similarly, in a neighbourhood of the point $a$,

$$
\begin{aligned}
& \tilde{B}^{(2)}(z)=-i \frac{5}{48} \frac{1}{h(b)}\left(\begin{array}{cc}
-1 & -i \\
-i & 1
\end{array}\right), \\
& \tilde{B}^{(1)}(z)=-\frac{i}{16} \frac{\frac{1}{2} h(a)+(a-b) h^{\prime}(a)}{(a-b) h^{2}(a)}\left(\begin{array}{cc}
1 & i \\
i & -1
\end{array}\right) \\
& +\frac{i}{16} \frac{1}{(a-b) h(a)}\left(\begin{array}{cc}
\left(d_{0}^{2}(a-b)^{2}-\frac{3}{2}\right) & i\left\{\frac{19}{6}+d_{0}^{2}(a-b)^{2}-4 d_{0}(a-b)\right\} \\
\left\{\frac{19}{6}+d_{0}^{2}(a-b)^{2}+4 d_{0}(a-b)\right\} & -\left(d_{0}^{2}(a-b)^{2}-\frac{3}{2}\right)
\end{array}\right) .
\end{aligned}
$$

We observe that for $c_{0}=0$, the expression for $\tilde{B}^{(1)}(z)$ is

$$
\tilde{B}^{(1)}(z)=-\frac{i}{16} \frac{\frac{1}{2} h(a)+(a-b) h^{\prime}(a)}{(a-b) h^{2}(a)}\left(\begin{array}{cc}
1 & i \\
i & -1
\end{array}\right)+\frac{i}{16} \frac{1}{(a-b) h(a)}\left(\begin{array}{cc}
-\frac{3}{2} & i \frac{19}{6} \\
i \frac{19}{6} & \frac{3}{2}
\end{array}\right) .
$$

Thus, outside neighbourhoods of the endpoints of the support, $r_{1}(z)$ can be written down in the following form:

$$
\begin{aligned}
r_{1}(z)= & \frac{\tilde{A}^{(2)}}{(z-b)^{2}}+\frac{\tilde{A}^{(1)}}{(z-b)} \frac{\tilde{B}^{(2)}}{(z-a)^{2}}+\frac{\tilde{B}^{(1)}}{(z-b)} \\
= & \frac{1}{(z-b)^{2}} \frac{5 i}{48 h(b)}\left(\begin{array}{cc}
-1 & i \\
i & 1
\end{array}\right) \\
& -\frac{1}{(z-b)}\left\{\frac{i}{16} \frac{\frac{1}{2} h(b)+(b-a) h^{\prime}(b)}{(b-a) h^{2}(b)}\left(\begin{array}{cc}
-1 & i \\
i & 1
\end{array}\right)\right. \\
& \quad \frac{i}{16(b-a) h(b)} \\
& \left.\times\left(\begin{array}{cc}
i\left\{\frac{19}{6}+c_{0}^{2}(b-a)^{2}+4 c_{0}(b-a)\right\} & i\left\{\frac{19}{6}+c_{0}^{2}(b-a)^{2}-4 c_{0}(b-a)\right\} \\
\left(c_{0}^{2}(b-a)^{2}-\frac{3}{2}\right)
\end{array}\right)\right\} \\
& -\frac{1}{(z-a)^{2}} \frac{5 i}{48 h(b)}\left(\begin{array}{cc}
-1 & -i \\
-i & 1
\end{array}\right)
\end{aligned}
$$




$$
\begin{aligned}
& -\frac{1}{(z-b)}\left\{\frac{i}{16} \frac{\frac{1}{2} h(a)+(a-b) h^{\prime}(a)}{(a-b) h^{2}(a)}\left(\begin{array}{cc}
1 & i \\
i & -1
\end{array}\right)\right. \\
& -\frac{i}{16} \frac{1}{(a-b) h(a)} \\
& \left.\times\left(\begin{array}{cc}
\left(d_{0}^{2}(a-b)^{2}-\frac{3}{2}\right) & i\left\{\frac{19}{6}+d_{0}^{2}(a-b)^{2}-4 d_{0}(a-b)\right\} \\
\left\{\frac{19}{6}+d_{0}^{2}(a-b)^{2}+4 d_{0}(a-b)\right\} & -\left(d_{0}^{2}(a-b)^{2}-\frac{3}{2}\right)
\end{array}\right)\right\},
\end{aligned}
$$

and formula (8), given in the Introduction, follows from this.

\section{EXAMPLES}

In this section we will use Theorem 1 to obtain asymptotic expansions for generalized Laguerre polynomials.

We define the generalized Laguerre polynomials by an exact formula that makes sense for every value $\alpha \in \mathbb{C}$ :

$$
L_{n}^{\alpha}(x):=\sum_{k=0}^{n}\left(\begin{array}{c}
n+\alpha \\
n-k
\end{array}\right) \frac{(-x)^{k}}{k !} .
$$

These polynomials can be also defined by the Rodrigues formula,

$$
L_{n}^{\alpha}(x)=\frac{1}{n !} x^{-\alpha} e^{x}\left(\frac{d}{d x}\right)^{n}\left[x^{\alpha+n} e^{-x}\right] .
$$

It is well known that for real $\alpha>-1$ the polynomials $L_{n}^{\alpha}$ are orthogonal on $[0, \infty)$ relative to the weight function $x^{\alpha} e^{-x}$ :

$$
\int_{0}^{\infty} L_{n}^{\alpha}(x) L_{m}^{\alpha}(x) x^{\alpha} e^{-x} d x=0 \quad \text { for } \quad n \neq m .
$$

Recently there has been much interest in the study of asymptotics of $\left\{L_{n}^{\alpha}\right\}$ when the exponent $\alpha$ depends on the index, that is, $\alpha:=\alpha_{n}$. In [27, the leading term of the asymptotic expansion was obtained for $\alpha=-A n$, where $A>1$. We consider a somewhat more general situation, $\alpha=-A n-A_{1}$, where $A_{1} \in \mathbb{R}$, and using Theorem 1 we obtain more exact asymptotic formulae.

Let us pass to compressed polynomials with leading coefficient equal to 1 :

$$
P_{n}^{\alpha}(z)=\frac{(-1)^{n} n !}{n^{n}} L_{n}^{\alpha}(n z), \quad n=0,1, \ldots .
$$

The orthogonality relations (102) become

$$
\int_{\Sigma} P_{n}^{\alpha}(z) z^{k} z^{\alpha} e^{-n z} d z \begin{cases}=0 & \text { for } k=0,1, \ldots, n-1, \\ \neq 0 & \text { for } k=n \text { and for } \alpha+n+1 \notin \mathbb{N},\end{cases}
$$

where $\Sigma$ is a Jordan arc in $\mathbb{C} \backslash[0, \infty)$ which is symmetric with respect to the real axis and such that

$$
\exists M>0 \forall x \geq M \exists y(x)>0: \Sigma \cap\{z: \mathfrak{R}(z)=x\}=\{x \pm i y(x)\},
$$

where in addition

$$
\exists \lim _{x \rightarrow \infty} y(x)=L<\infty
$$



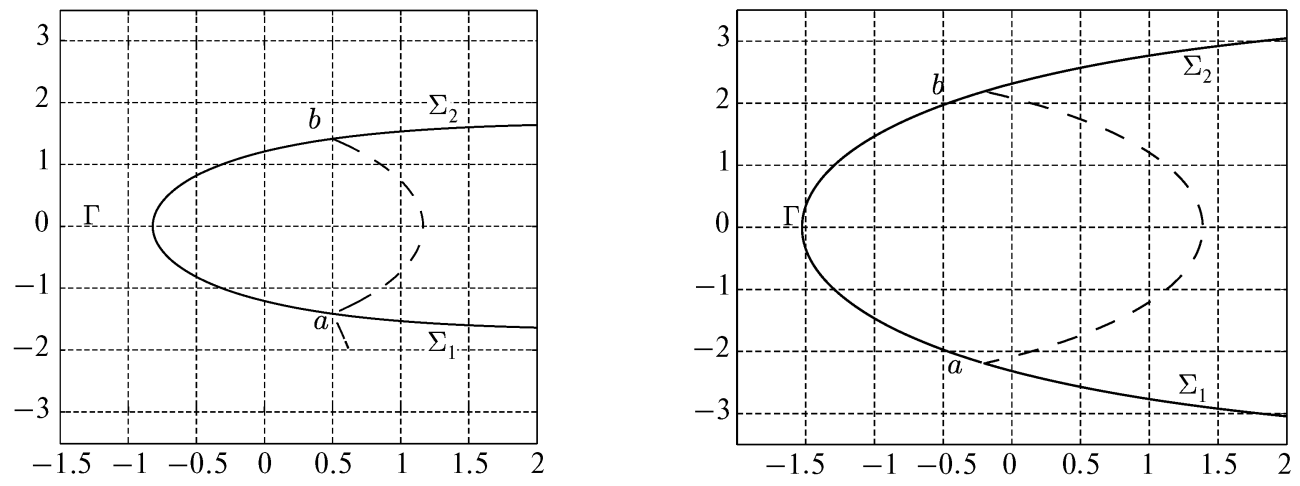

Figure 6. The contour $\Sigma$ for $A=1.5$ (left) and for $A=2.2$ (right)

From (104) we have that

$$
Q_{0}=\frac{1}{2}(z+A \ln z), \quad Q_{1}=\frac{1}{2} A_{1} \ln z .
$$

To apply Theorem 1, the contour $\Sigma$ must satisfy the symmetry condition (4) (the $S$-property). In 27] it is shown that $\Sigma=\Gamma \cup \Sigma_{1} \cup \Sigma_{2}$, where $\Gamma$ is an arc that connects $b=2-A+2 i \sqrt{A-1}$ with $a=\bar{b}$, and such that

$$
\frac{1}{2 \pi i} \int_{\bar{b}}^{z} \frac{\sqrt{(s-b)(s-\bar{b})}}{s} d s
$$

takes real values for all $z \in \Gamma$, while the $\operatorname{arcs} \Sigma_{1}$ and $\Sigma_{2}$ are such that in a neighbourhood of the points $b$ and $\bar{b}$ the values of

$$
\int_{\bar{b}}^{z} \frac{\sqrt{(s-b)(s-\bar{b})}}{s} d s, \quad \int_{b}^{z} \frac{\sqrt{(s-b)(s-\bar{b})}}{s} d s
$$

are, respectively, real and positive. Figure 6 shows examples of $\Sigma$ for two values of $A$.

In [27] it is shown that the support of the equilibrium measure in the field

$$
f(x):=\frac{1}{2}(z+A \ln z)
$$

is an $\operatorname{arc} \Gamma$ with endpoints at $b$ and $\bar{b}$, while the derivative of the equilibrium measure has the form

$$
\lambda^{\prime}(z)=\frac{\sqrt{(z-b)(z-\bar{b})}}{2 \pi i z} .
$$

To see that, we need first to verify that the complex measure

$$
\frac{1}{2 \pi i} \frac{\sqrt{(s-a)(s-b)}}{s} d s
$$

is a probability measure on $\Gamma$ and, second, that for $z \in \mathbb{C} \backslash\left\{\Gamma \cup \Sigma_{1} \cup[0, \infty)\right\}$ we have the following representation for the complex potential:

$$
\mathcal{V}_{\lambda}(z):=\mathcal{V}_{\lambda}(b)+\frac{1}{2}(A \ln (b)+b)-\frac{1}{2}(A \log z+z)+\frac{1}{2} \int_{b}^{z} \frac{\sqrt{(s-a)(s-b)}}{s} d s .
$$


From this, in particular, follows the equilibrium condition for the complex potential:

$$
\mathcal{V}_{\lambda+}(z)+\mathcal{V}_{\lambda-}(z)=-(A \ln (z)+z)+2\left(\mathcal{V}_{\lambda}(b)+\frac{1}{2}(A \ln (b)+b)\right)
$$

From (106), (107), and (18) it follows that

$$
\begin{aligned}
\varphi_{n}(z)= & \exp \left\{\frac{n}{2}(A \log z+z)-\frac{n}{2} \int_{b}^{z} \frac{\sqrt{(s-a)(s-b)}}{s} d s\right\} \\
& \times \exp \left\{\frac{((z-a)(z-b))^{1 / 2}}{2 \pi i} \int_{\Gamma} \frac{-A_{1} \ln (t)}{z-t} \frac{d t}{\sqrt{(t-a)(t-b)}}\right\} .
\end{aligned}
$$

For brevity, we restrict ourselves to the case $A=2$. Then (108) becomes

$$
\begin{aligned}
\varphi_{n}(z)= & \exp \left\{\frac{n}{2}(2 \log z+z)-\frac{n}{2} \int_{2 i}^{z} \frac{\sqrt{s^{2}+4}}{s} d s\right\} \\
& \times \exp \left\{\frac{\left(z^{2}+4\right)^{1 / 2}}{2 \pi i} \int_{\Gamma} \frac{-A_{1} \ln (t)}{z-t} \frac{d t}{\sqrt{t^{2}+4}}\right\} \\
= & e^{\pi i n / 2} \exp \left\{\frac{n}{2}\left(z-\sqrt{z^{2}+4}\right)\right\}\left(2+\sqrt{z^{2}+4}\right)^{n} \\
& \times \exp \left\{\frac{\left(z^{2}+4\right)^{1 / 2}}{2 \pi i} \int_{\Gamma} \frac{-A_{1} \ln (t)}{z-t} \frac{d t}{\sqrt{t^{2}+4}}\right\} .
\end{aligned}
$$

As $z \rightarrow \infty$,

$$
\varphi_{n}(z) \sim e^{\pi i n / 2} \exp \left\{\int_{\Gamma} \frac{-A_{1} \ln (t) d t}{\sqrt{t^{2}+4}}\right\} z^{n} \sim e^{\pi i n / 2} z^{n}
$$

and therefore

$$
C_{n}=e^{-\pi i n / 2}
$$

Since for $A=2$

$$
a=-2 i, \quad b=2 i, \quad h(x)=\frac{1}{2 \pi i x},
$$

substituting these values into (8), we obtain

$$
\Pi_{1}(z)=\frac{5 \pi i\left(z^{2}-4\right)}{6\left(z^{2}+4\right)^{2}}+\pi c_{0}^{2}-\pi d_{0}^{2}+\pi \frac{z-\sqrt{z^{2}+4}}{z}\left(c_{0}^{2}+d_{0}^{2}-2 i \frac{3 z^{2}+2}{3\left(z^{2}+4\right)^{2}}\right),
$$

while for $c_{0}$ and $d_{0}$ we have

$$
c_{0}:=\frac{2}{\pi i} \int_{\Gamma} \frac{-A_{1} \ln \zeta}{((\zeta-a)(\zeta-b))^{1 / 2}} \frac{d \zeta}{(\zeta-b)}
$$

and

$$
d_{0}:=\frac{2}{\pi i} \int_{\Gamma} \frac{-A_{1} \ln \zeta}{((\zeta-a)(\zeta-b))^{1 / 2}} \frac{d \zeta}{(\zeta-a)} .
$$

Finally, substituting (109), (110), (111) into (6), we see that for the compressed Laguerre polynomials $P_{n}^{\alpha}, \alpha=-2 n-A_{1}<-1, A>1$ (see (103)), uniformly on compact sets in $\mathbb{C} \backslash \Gamma$, we have the following asymptotic formula as $n \rightarrow \infty$ :

$$
\begin{aligned}
P_{n}^{\alpha}(z)= & \exp \left\{\frac{n}{2}\left(z-\sqrt{z^{2}+4}\right)\right\}\left(2+\sqrt{z^{2}+4}\right)^{n} \\
& \times \exp \left\{\left(z^{2}+4\right)^{1 / 2} \int_{\Gamma} \frac{-A_{1} \ln (t)}{z-t} \frac{d t}{2 \pi i \sqrt{t^{2}+4}}\right\}\left(\frac{\sqrt{z-2 i}+\sqrt{z+2 i}}{2\left(z^{2}+4\right)^{1 / 4}}\right)
\end{aligned}
$$




$$
\begin{aligned}
& \times\left[1+\left(\frac{5 i\left(z^{2}-4\right)}{6\left(z^{2}+4\right)^{2}}+c_{0}^{2}-d_{0}^{2}\right.\right. \\
& \left.\left.\quad+\frac{z-\sqrt{z^{2}+4}}{z}\left(c_{0}^{2}+d_{0}^{2}-2 i \frac{3 z^{2}+2}{3\left(z^{2}+4\right)^{2}}\right)\right) \frac{\pi}{n}+O\left(\frac{1}{n^{2}}\right)\right] .
\end{aligned}
$$

\section{REFERENCES}

[1] A. A. Gonchar and E. A. Rakhmanov, Equilibrium distributions and rate of convergence of rational approximation of analytic functions, Mat. Sbornik 134 (1987), no. 3, 306-352; English transl. in Math. USSR Sb. 62 (1989), no. 2, 305-348. MR0922628

[2] A. I. Aptekarev, Sharp constants for rational approximations of analytic functions, Mat. Sbornik 193 (2002), no. 1, 3-72; English transl. in Sb. Math. 193 (2002), no. 1-2, 1-72. MR.1906170

[3] P. Deift, T. Kriecherbauer, K. T.-R. McLaughlin, S. Venakides and X. Zhou, Uniform asymptotics for polynomials orthogonal with respect to varying exponential weights and applications to universality question in random matrix theory, Comm. Pure Appl. Math. 52 (1999), no. 11, 1335-1425. MR.1702716

[4] P. Bleher and A. Its, Semiclassical asymptotics of orthogonal polynomials, Riemann-Hilbert problem and universality in the matrix model, Math. Ann. 150 (1999), no. 2, 185-266. MR.1715324

[5] L. Pastur and M. Shcherbina, Universality of the local eigenvalue statistics for a class of unitary invariant matrix ensembles, J. Stat. Phys. 6 (1997), 109-147. MR.1435193

[6] J. Baik, K. T-R. McLaughlin, P. Miller and X. Zhou, Optimal tail estimates for directed last passage site percolation with geometric random variables, Adv. Theor. Math. Phys. 5 (2001), 1207-1250. MR 1926668

[7] J. Baik, P. Deift and K. Johansson, On the distribution of the length of the longest increasing subsequence of random permutations, J. Amer. Math. Soc. 12 (1999) 1119-1178. MR.1682248

[8] A. Fokas, A. Its and A. Kitaev, The isomonodromy approach to matrix models in $2 D$ quantum gravity, Commun. Math. Phys. 147 (1992), 395-430. MR1174420

[9] S. Kamvissis, K. McLauhglin and P. Miller, Semiclassical soliton ensembles for the focusing nonlinear Schrödinger equation, Annals of Mathematics Studies, 154, Princeton Univesity Press, Princeton, NJ, 2003. MR1999840

[10] S. N. Bernstein, Sur les polynômes orthogonaux relatifs à un segment fini, I: J. Math. Pures Appl. 9 (1930), 127-177; II: J. Math. Pures Appl. 10 (1931), 219-286.

[11] G. Szegö, Orthogonal polynomials, AMS Colloquium Publications, vol. 23, Revised ed., AMS, Providence, RI, 1959. MR.0106295

[12] J. Nutall and S. R. Singh, Orthogonal polynomials and Padé approximants associated with a system of arcs, J. Approx. Theory 21 (1977), 1-42. MR0487173

[13] J. Nuttall, Asymptotics of diagonal Hermite-Padé polynomials, J. Approx. Theory 42 (1984), no. 4, 299-386. MR0769985

[14] J. Nuttall, Padé polynomial asymptotics from a singular integral equation, Constr. Approx. 6 (1990), no. 2, 157-166. MR1036606

[15] S. P. Suetin, On uniform convergence of diagonal Padé approximants for hyperelliptic functions, Mat. Sbornik 191 (2000), no. 9, 81-114; English transl. in Sb. Math. 191 (2000), no. 9-10, 13391373. MR 1805599

[16] H. Stahl, Orthogonal polynomials with complex valued function. I, II, Constr. Approx. 2 (1986), 225-240, 241-251. MR0891973

[17] A. R. Its, A. V. Kitaev and A. S. Fokas, An isomonodromy approach to the theory of two-dimensional quantum gravitation, Uspekhi Mat. Nauk 45 (1990), no. 6, 135-136; English transl. Russian Math. Surveys 45 (1990), no. 6, 155-157. MR1101341 (92c:81166)

[18] P. Deift and X. Zhou, A steepest descent method for oscillatory Riemann-Hilbert problems: asymptotics for the MKdV equation, Ann. of Math. 137 (1993), 295-368. MR.1207209

[19] P. Deift, T. Kriecherbauer, K. T.-R. McLaughlin, S. Venakides and X. Zhou, Asymptotics for polynomials orthogonal with respect to varying exponential weights, Int. Math. Res. Notes 16 (1997), 759-782. MR:1472344

[20] P. Deift, T. Kriecherbauer, K. T.-R. McLaughlin, S. Venakides and X. Zhou, Strong asymptotics of orthogonal polynomials with respect to exponential weights, Comm. Pure Appl. Math. LII (1999), 1491-1552. MR:1711036

[21] P. Deift, T. Kriecherbauer, K. T.-R.McLaughlin, S. Venakides and X. Zhou, Uniform asymptotics for orthogonal polynomials, Doc. Math. J. DMV, Extra Vol. ICM Berlin 3 (1998), 491-501. MR1648182 
[22] P. Deift, Orthogonal polynomials and random matrices: a Riemann-Hilbert approach, Courant Lecture Notes in Mathematics, 3. New York University, Courant Institute of Mathematical Sciences, New York; American Mathematical Society, Providence, RI, 1999. MR.1677884

[23] A. I. Aptekarev and W. Van Assche, Scalar and matrix Riemann-Hilbert approach to the strong asymptotics of Padé approximants and complex orthogonal polynomials with varying weight, J. Approx. Theory 129 (2004), no. 2, 129-166. MR2078646

[24] N. M. Ercolani and K. T.-R. McLaughlin, Asymptotics of the partition function for random matrices via Riemann-Hilbert techniques, and applications to graphical enumeration, Int. Math. Res. Not. 2003, no. 14, 755-820. MR 1953782

[25] A. B. J. Kuijlaars, K. T.-R. Mclaughlin, W. Van Assche, and M. Vanlessen, The Riemann-Hilbert approach to strong asymptotics for orthogonal polynomials on $[-1,1]$, Adv. Math. 188 (2004), 337-398. MR2087231

[26] R. F. Khabibullin, Asymptotic series for Bessel polynomials, Mat. Zametki 77 (2005), no. 6, 948950; English transl., Math. Notes 77 (2005), no. 5-6, 878-881. MR2246973

[27] A. B. J. Kuijlaars and K. T.-R. McLaughlin, Riemann-Hilbert analysis for Laguerre polynomials with a large negative parameter, Comput. Meth. Funct. Theory. 1 (2001), 205-233. MR 1931612

[28] F. D. Gakhov, Boundary value problems, Fizmatgiz, Moscow 1963; English transl. (2nd ed.), Pergamon Press, Oxford, 1966. MR0104117 MR0198152

[29] M. Abramowitz and I. Stegun, eds., Handbook of mathematical functions: with formulas, graphs, and mathematical tables, Dover, New York, 1966. MR0208797

The M. V. Keldysh Applied Mathematics Institute, Russian Academy of Sciences, Miusskaya SQ. 4, Moscow 125047, Russia

E-mail address: aptekaa@keldysh.ru

The M. V. Keldysh Applied Mathematics Institute, Russian Academy of Sciences, Miusskaya SQ. 4, Moscow 125047, Russia

Translated by MICHAEL GRINFELD 